\title{
The Properties of Typhoon-Scale Disturbances Produced by Arakawa-Schubert Parameterization
}

\author{
By Misuzu Wada \\ Meteorological Research Institute, Tokyo \\ (Manuscript received 19 October 1976, in revised form 20 May 1977)
}

\begin{abstract}
In order to obtain a theoretical base of formation and development of a tropical cyclone, properties of unstable waves produced by cumulus convection which is expressed by a parameterization scheme proposed by Arakawa and Schubert (1974) are investigated by a linear analysis.

The model used is a four-layer model containing three layers of the free atmosphere and one mixed layer. Thus three cloud types such as the highest type $(\mathrm{H})$, the middle type (M) and the lowest type (L) are defined. The following seven combinations may be considered; (HML), (HM), (HL), (ML), (H), (M) and (L), depending on the co-existence of these cloud types.

Among these seven, the combination which is accompanied by a large scale disturbance is uniquely determined as an eigen solution for almost all cases if the basic field and the scale of the disturbance are specified. In general, the mode $(\mathrm{H})$, the mode $(\mathrm{HM})$ and the mode (HML) appear in turn with increasing wavelength. The mode which becomes most unstable depends on the vertical distribution of temperature and water vapour of the basic field.

The typhoon-scale disturbance whose preferred scale is less than a few hundred kilometers and growth rate is in a range of $10^{-6} \sim 10^{-5} \mathrm{sec}^{-1}$ belongs to eigher the mode $(\mathrm{H})$ or the mode (HM). These modes can be unstable as the lower layer of basic field becomes conditionally unstable and humid. The convective heating in the lower layer plays an essential role for the typhoon-scale disturbance to become unstable.

Finally, a comparison is attempted between the typhoon-scale disturbance mentioned above and the typhoon mode of Ooyama (1964).
\end{abstract}

\section{Introduction}

The interaction between large scale disturbance and cumulus clouds embeded in it is one of the most important subjects in the tropical meteorology. Since the concept of the conditional instability of the second kind (CISK) was established by Ooyama (1964) and Charney and Eliassen (1964), many investigations on a tropical cyclone have been attempted by Ooyama (1969), Kuo (1965), Syono and Yamasaki (1966), Yamasaki (1968 a, b, c), Rosenthal (1970, 1973), Kurihara and Tuleya (1964), Kurihara (1975) and others.

The parameterization schemes used in these investigations are, roughly speaking, divided into following two kinds; one is convective adjustment and the other is penetrative convection model. Convective adjustment has been used in general circulation model by Manabe et al. (1965). Recently a sophisticated version of the param- eterization of this kind was presented by Kurihara (1974) and was used in numerical experiments of a tropical cyclone by Kurihara and Tuleya (1974, 1975) and Kurihara (1975). On the other hand, the penetrative convection model was originally proposed by Ooyama (1964). As well known, the parameterization is based on the assumption that the convective heating in the free atmosphere is proportional to large scale upward motion produced by the surface frictional convergence. Ooyama (1969) succeeded in simulation of essential features of a tropical cyclone using the parameterization.

Syono and Yamasaki (1966) and Yamasaki $(1968,1969)$ pointed out that the vertical profile of the convective heating is an important factor to development and the structure of the disturbance. It was revealed that the convective heating in the lower part of the troposphere is essential for typhoon-scale disturbance to develop. How- 
ever, whether or not the vertical profile of the heating parameter used in these investigations is realistic as a representation of cumulus activity, has been an unsolved question. Recently, Ooyama (1971) attempted to determine the vertical profile of the heating parameter more physically using a one-dimensional steady state cloud model. However, in his proposal, the cloud mass flux at the cloud base level is given by a kind of size distribution function of cloud elements, so-called 'dispatcher function' and a question how to relate the cloud mass flux with variables of the large scale disturbance under consideration, remained unsolved. In this sense, this parameterization is yet an unclosed scheme too.

Arakawa and Schubert (1974) attempted to relate the cloud mass flux, which is essentially the same as the dispatcher function of Ooyama, with variables of large scale disturbances. At first 'cloud work function' which is a measure of the kinetic energy generation of cumulus cloud, is defined. The cloud work function may change with time both by forcing of large scale motion and by effects of cumulus cloud. However, if the time scale of large scale forcing is much longer than the adjustment time of clouds which is about $10^{3} \sim 10^{4} \mathrm{sec}$, it is hypothesized that the cumulus ensemble will follow a sequence of quasiequilibrium states with the large scale forcing. Then the cloud mass flux is determined in such a way that the quasi-equilibrium assumption on the cloud work function is satisfied. The parameterization is considered to be the most promising one at present.

The purpose of the present study is to examine whether or not a typhoon-like disturbance can be obtained by the parameterization proposed by Arakawa and Schubert (1974), and to reveal the conditions of basic fields which are favorable to development and formation of such a disturbance. For this purpose we investigate properties of unstable waves by linear analysis.

In sections 2 and 3, the formulation of convective heating based on the parameterization in a linear model is described. The convective heating is expressed by a linear function of vertical motion of large scale disturbance not only at the cloud base but also at all levels. This is an important difference between the parameterization of Arakawa and Schubert (1974) and that of Ooyama (1964). The stability properties of waves obtained by the present linear analysis are described in sections 4,5 and 6 . The uniqueness of solution is investigated in section 4 . The effects of basic field are described in section 5 and the structures in section 6 . In section 7 , a comparison will be made between typhoon-like disturbance obtained by the present analysis and typhoon mode of Ooyama $(1964,1969)$ and Syono and Yamasaki (1969).

\section{The basic assumptions of the linear model}

\subsection{Perturbation equation}

The model used in the present study is the two-dimensional primitive equation system with the $Z$-coordinate in the vertical direction. The linearized system of the basic equations may be written as follows.

$$
\begin{aligned}
& \overline{\bar{\rho}} \frac{\partial \bar{u}}{\partial t}=\overline{\bar{\rho}} f \bar{v}-\frac{\partial \bar{p}}{\partial x}+\frac{\partial \tau_{x}}{\partial z} \\
& \overline{\bar{\rho}} \frac{\partial \bar{v}}{\partial t}=-\overline{\bar{\rho}} f \bar{u}+\frac{\partial \tau_{y}}{\partial z} \\
& \overline{\bar{\rho}} \frac{\partial \bar{S}}{\partial t}=-\overline{\bar{\rho}} \frac{\partial \bar{S}}{\partial z} \bar{w}+Q c \\
& \overline{\bar{\rho}} \frac{\partial \bar{u}}{\partial x}=-\frac{\partial \overline{\bar{\rho}} \bar{w}}{\partial z} \\
& \frac{\partial \bar{p}}{\partial z}=-g(\overline{\bar{\rho}} / \overline{\bar{p}})\left(\bar{p}-R \overline{\bar{\rho}} \bar{S} / C_{p}\right)
\end{aligned}
$$

Notations used here are; $u=$ component of horizontal wind in the $X$-direction, $v=$ component of horizontal wind in the $Y$-direction, $w=$ vertical wind velocity, $p=$ pressure, $S=$ static energy ( $=C_{p} T+g Z$ ), $\rho=$ density of the air, $R=$ gas constant of the air, $C_{p}=$ specific heat of the air at a constant pressure, $g=$ the acceleration of gravity, $f=$ Coriolis parameter, $\tau_{x}=X$-component of vertical shear stress, $\tau_{y}=Y$-component of vertical shear stress, $Q_{c}=$ diabatic heating by parameterized cumulus convection. Over-bars (-) denote variables of the large scale disturbance under consideration and double-bars $(=)$ those of the basic field which are functions of $z$ only.

Eq. (2.1) and Eq. (2.3) are equations of motion in the $X$ - and $Y$-direction respectively. Eq. (2.3) is the first law of thermodynamics. Eq. (2.4) is the equation of continuity and Eq. (2.5) is the hydrostatic relation.

Internal friction is not included in the present study, and only the surface stress is taken into consideration using the following simplified form.

$$
\begin{aligned}
\tau_{x s} & =\bar{p}_{s} D_{f} \bar{u}_{s} \Delta z \\
\tau_{z s} & =\overline{\bar{\rho}}_{s} D_{f} \bar{v}_{s} \Delta z
\end{aligned}
$$

where

$$
D_{f}=C_{D}\left|V_{s}\right| / \Delta z
$$

Here, $C_{D}$ is the drag coefficient and $V_{S}$ the total 
surface wind, $\Delta z$ the depth of friction layer. The subscript $s$ denotes a value at the surface. In the present study, $D_{f}$ is $10^{-5} \mathrm{sec}^{-1}$ and $f$ is $5 \times 10^{-5}$ $\sec ^{-1}$.

Our problem is to solve the set of equation system from (2.1) to (2.5) under the following boundary condition,

$$
w=0 \text { at } z=0 \text { and } z=z_{T},
$$

where $z_{T}$ is the height of the upper boundary of the model atmosphere.

\subsection{The derivation of convective heating func-} tion

In order to investigate the properties of a large scale disturbance driven by parameterized cumulus convection as an eigen value problem we express $Q_{c}$ in Eq. (2.3) as a linear function of variables of the large scale disturbance. In the present section, we shall describe a formulation of heating function that is based on the Arakawa-Schubert theory.

Introducing the concept of statistical steadiness of cumulus clouds, Arakawa (1969) and Ooyama (1971) made it clear that cumulus clouds change the large scale temperature and moisture fields both by warming and drying due to cumulusinduced subsidence and by moistening due to detrainment of water content from clouds to the environment. The linearized representation of time change of static energy of large scale field is given as follows.

$$
\overline{\bar{\rho}} \frac{\partial \bar{S}}{\partial t}=D\left(\hat{S}_{c}-\overline{\bar{S}}\right)-L D l_{c}+\frac{\partial \overline{\bar{S}}}{\partial z} M_{c}-\frac{\partial \overline{\bar{S}}}{\partial z} \overline{\bar{\rho}} w
$$

Following the above mentioned authors, we consider co-existing of several cloud types. For each type, terminal detrainment is assumed at the cloud top level, $Z_{D}(\lambda) . \quad \lambda$ is entrainment rate and fully characterizes a cloud type. The heating function in Eq. (2.3) corresponds to the first three terms of the right hand side of Eq. (2.10), that is

$$
\begin{aligned}
Q_{c}(z)= & D(z)\left(\hat{S}_{c}\left(\lambda_{D}(z)\right)-\overline{\bar{S}}(z)\right) \\
& -L D(z) \hat{l}_{c}\left(\lambda_{D}(z)\right)+\frac{\partial \overline{\bar{S}}(z)}{\partial z} M_{c}(z)
\end{aligned}
$$

where $\lambda_{D}(z)$ is the entrainment rate of a cloud type whose top is at the level $Z$. The subscript $(C)$ denotes values in clouds and a hat $(\wedge)$ those at cloud top level. $l$ is mixing ratio of liquid water content. $M_{c}(Z)$ is the sum of vertical cloud mass flux of all types of clouds at the level $Z$ and $D(Z)$ is detraining mass flux from cloud top to the environment at the level $Z$.
If a basic field and a cloud base condition are given, thermal properties in clouds are estimated for each type by using a cloud model which will be described in the sub-section (3.2). $M_{c}(Z)$ and $D(Z)$ are determined if cloud mass flux of each type at the cloud base level, $m_{B}(\lambda)$ is known. Therefore, the only one unknown quantity in Eq. (2.11) is $m_{B}(\lambda)$, which is essentially the same as 'dispatcher function' of Ooyama (1971).

Arakawa and Schubert (1974) proposed 'quasiequilibrium assumption' for determination of $m_{B}(\lambda)$. Cloud work function of $\lambda$-type, $A(\lambda)$, may increase with time by cooling and moistening effects of large scale upward motion or may decrease by warming and drying effect of cumulus-induced subsidence by all types of clouds including the $\lambda$-type itself.

$$
\frac{\partial A(\lambda)}{\partial t}=\int_{0}^{\lambda_{\max }} K\left(\lambda, \lambda^{\prime}\right) m_{B}\left(\lambda^{\prime}\right) d \lambda^{\prime}+F(\lambda)
$$

where the first term on the right hand side of Eq. (2.12) is the effect of $\lambda^{\prime}$-type clouds upon the time change of the cloud work function of $\lambda$-type, while the second term expresses the effect of large scale forcing.

To simplify above equation, we adopt following assumptions. (1) The time change of thermal properties in the mixed layer such as $S_{M}, q_{M}$, $h_{M}$ and $l_{M}$ are not taken into consideration. (2) We assume that height of cloud base, $Z_{B}$, coincides with the condensation level, $Z_{c}(\lambda)$, for all types and neglect the difference in $Z_{c}(\lambda)$ for each type. (3) We neglect effect of liquid water upon the air density. Thus virtual static energy, $S_{v}$, is approximated by

$$
S_{v}=C_{p} T^{*}+g z
$$

where $T^{*}$ is virtual temperature. The first assumption is indispensable for the formulation of $Q_{c}$ by a linear function of variables of the large scale disturbance while the second and the third ones are for mathematical simplicity.

Using above assumptions, we obtain the following simplified form of $\partial A(\lambda) / \partial t$.

$$
\begin{aligned}
\frac{\partial A(\lambda)}{\partial t}= & \int_{0}^{\lambda_{\max }} K\left(\lambda, \lambda^{\prime}\right) m_{B}\left(\lambda^{\prime}\right) d \lambda^{\prime} \\
& -\int_{Z_{B}}^{Z_{D}(\lambda)} F_{c}(z, \lambda) \overline{\bar{\rho}}(z) \bar{w}(z) d Z \\
& -K_{\mathrm{MIX}}(\lambda) \overline{\bar{\rho}}\left(Z_{B}\right) \bar{w}\left(Z_{B}\right)
\end{aligned}
$$

where

$$
\int_{0}^{\lambda_{\max }} K\left(\lambda, \lambda^{\prime}\right) m_{B}\left(\lambda^{\prime}\right) d \lambda^{\prime}
$$




$$
\begin{aligned}
= & \int_{\lambda}^{\lambda_{\max }} K_{D}\left(\lambda \cdot \lambda^{\prime}\right) m_{B}\left(\lambda^{\prime}\right) d \lambda^{\prime} \\
& +\int_{0}^{\lambda_{\max }} K_{v}\left(\lambda, \lambda^{\prime}\right) m_{b}\left(\lambda^{\prime}\right) d \lambda^{\prime} \\
& +\int_{0}^{\lambda_{\max }} K_{\mathrm{MIX}}(\lambda) m_{B}\left(\lambda^{\prime}\right) d \lambda^{\prime}
\end{aligned}
$$

A detailed derivation is described in Appendix (A).

Quasi-equilibrium assumption can be written as follows.

$$
\begin{array}{ll}
m_{B}(\lambda)>0 \text { and } & \int_{0}^{\lambda_{\max }} K\left(\lambda, \lambda^{\prime}\right) m_{B}\left(\lambda^{\prime}\right) d \lambda^{\prime} \\
+F(\lambda)=0 & \\
m_{B}(\lambda)=0 \text { and } & \int_{0}^{\lambda_{\max }} K\left(\lambda, \lambda^{\prime}\right) m_{B}\left(\lambda^{\prime}\right) d \lambda^{\prime} \\
+F(\lambda)<0 &
\end{array}
$$

If the adjustment time of clouds is small enough in comparison with the time scale of the large scale disturbance under consideration, we may assume that the ensemble of cumulus clouds follows a sequence of quasi-equilibrium states as the large scale forcing slowly changes, and the upper condition of (2.15) must hold for each type whose $m_{B}(\lambda)$ is positive. However, when the net increase of $A(\lambda)$ by the large scale forcing plus the forcing by other types is negative, $\lambda$-type may not exist because the $\lambda$-type clouds can not increase its own $A(\lambda)$ and the lower condition of (2.15) must hold for each type whose $m_{B}(\lambda)$ is zero.

Our problem is how to relate $m_{B}(\lambda)$ with vertical motion of large scale disturbances. If we know what types exist, $m_{B}(\lambda)$ can then be expressed by a linear function of large scale vertical motion, $\overline{\bar{\rho}} \bar{w}$, by applying the upper condition of (2.15). However, we do not know what types exist a priori. It is a problem that should be solved. In this sense, our system which includes the condition (2.15), is essentially non-linear.

In the present analysis, we solve the problem as follows. At first, we consider a combination of cloud types in which some types are assumed to exist and others not to exist. Hereafter, the type for which the condition that the time derivative of $A(\lambda)$ vanish is required, is referred to as the existing type and the type whose $m_{B}(\lambda)$ is assumed to be zero, as the suppressed type. If we consider a certain combination, each $m_{B}(\lambda)$ of the former type is expressed as functions of $\rho w$ and the equation system from (2.1) to (2.5) can be solved as an ordinary eigen value problem. However, a set of eigen solutions thus obtained does not necessarily satisfy the condition
(2.15). Only when all of the existing type included in the combination have positive $m_{B}(\lambda)$ and all $A(\lambda)$ of the suppressed type decrease with time, the set of eigen solution can be recognized as a solution that satisfies quasi-equilibrium assumption. As we do not know what combinations produce such solution in advance, we solve a set of eigen value problems for all combinations which can be possible in the model layer configuration. Then, we chose the solutions that satisfy the condition (2.15). Detailes are described in the following section. Further it is an important point whether or not the combination which produces the solution satisfying the condition (2.15), is uniquely determined. The uniqueness of solution is investigated in section 4 .

In the present linear model, unconditional heating is assumed for mathematical simplicity. Therefore, negative heating due to negative cloud mass flux occurs in the region of large scale downward motion. However, stability properties of stationary waves which are discussed in the present study, may not be significantly different between the assumption of unconditional and conditional heating (c.f. Syono and Yamasaki, 1966).

In solutions of Eq. (2.1)-(2.5) some propagating waves are included besides stationary waves. A basic difficulty in methodology is involved in the treatment of propagating waves. As mentioned above, we distinguish the self-consistent solutions from others by applying the condition (2.15), after solving eigen values problems for all combinations. Because $m_{B}(\lambda)$ of each type and $\overline{\bar{\rho}} \bar{w}$ at each level obtained as eigen solutions generally take complex values in propagating waves, that is, there exist phase differences between these quantities, we can not judge whether the solutions satisfy the quasi-equilibrium assumption or not. For this reason we will not deal with the propagating waves in the present linear analysis. We shall confine ourselves to investigation of stationary unstable disturbances. Under some conditions propagating disturbances may become solutions that satisfy the quasi-equilibrium assumption if we assume the conditional heating. In this respect, the present paper can not be regarded as a full analysis of disturbances which are possible under Arakawa-Schubert parameterization.

\section{The finite difference representation of the model}

\subsection{Model for large scale disturbances}

We shall solve equation system from (2.1) to 


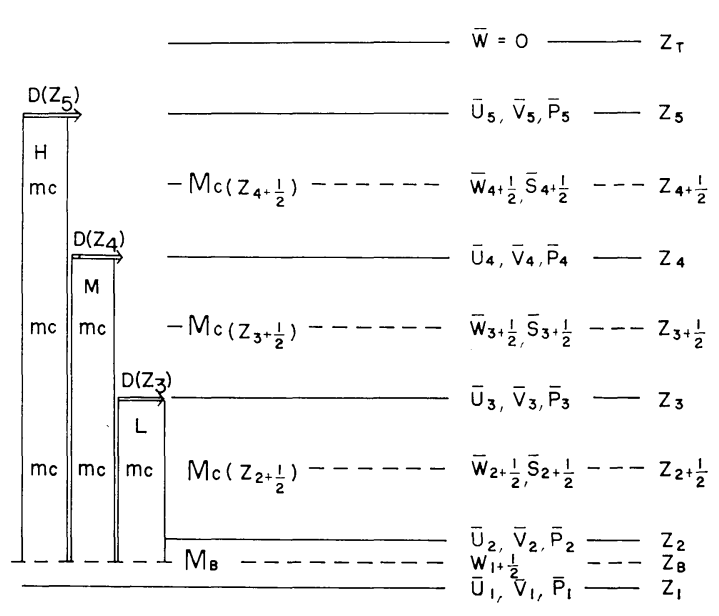

Fig. 1 Four-layer model and three cloud-types.

(2.5) numerically by finite difference method. We shall use a four-layer model as shown in Fig. 1. Upper three layers correspond to the free atmosphere and the lowest one to the mixed layer.

Let the five dependent variables $(\bar{u}, \bar{v}, \tilde{w}, \tilde{s}, \bar{q})$ be defined at discrete levels as shown in Fig. 1. The equation of motion is applied at levels $Z=$ $Z_{i}(i=1,2,3,4,5)$ and the thermodynamic equation at the mid-levels between $Z_{i}$ and $Z_{i+1}(i=$ $2,3,4)$ in the free atmosphere. The time change of static energy in the mixed layer is not taken into consideration where the neutral stratification $(\partial \bar{S} / \partial Z=0)$ is assumed.

Using the assumption of unconditional heating, we may consider a sinusoidal wave in the zonal direction $(\partial / \partial x=i k)$ and the time dependency of disturbance as $\partial / \partial t=-i k C$. Then Eq. (2.1) to (2.5) are transformed into

$$
\begin{gathered}
-i k C \overline{\bar{\rho}}_{i} \tilde{u}_{i}-f \overline{\bar{\rho}}_{i} \tilde{v}+i k \tilde{p}_{i}=0 \\
(i=2,3,4,5) \\
-i k C \overline{\bar{\rho}}_{1} \tilde{u}_{1}-f \overline{\bar{\rho}}_{1} \tilde{v}_{1}+i k \tilde{p}_{1}+\overline{\bar{\rho}}_{1} D_{f} u_{1}= \\
-i k C \bar{\rho}_{i} \tilde{v}_{i}+f \overline{\bar{\rho}}_{i} \tilde{u}_{i}-0 \quad(i=2,3,4,5) \\
-i k C \overline{\bar{\rho}}_{1} \tilde{v}_{1}+f \overline{\bar{\rho}}_{1} \tilde{u}_{1}+\rho_{1} D_{\rho} \tilde{v}_{1}=0 \\
-i k C \overline{\bar{\rho}}_{i+(1 / 2)} \tilde{S}_{i+(1 / 2)}+\overline{\bar{\rho}}_{i+(1 / 2)} \\
\frac{\partial \overline{\bar{S}}}{\partial Z} \tilde{w}_{i+(1 / 2)}-Q_{c}\left(Z_{i+(1 / 2)}\right)=0 \\
i+(1 / 2) \\
\tilde{S}_{1+(1 / 2)}=0 \quad(i=2,3,4) \\
i k \overline{\bar{\rho}}_{i} \tilde{u}_{i}+\left(\overline{\bar{\rho}}_{i+(1 / 2)} \tilde{w}_{i+(1 / 2)}\right. \\
\left.\quad-\overline{\bar{\rho}}_{i-(1 / 2)} \tilde{w}_{i-(1 / 2)}\right) /\left(Z_{i+(1 / 2)}\right. \\
\left.\quad-Z_{i-(1 / 2)}\right) \quad(i=2,3,4,5) \\
i k \overline{\bar{\rho}}_{1} \tilde{u}_{1}+\left(\overline{\bar{\rho}}_{1+(1 / 2)} \tilde{w}_{1+(1 / 2)}\right) / Z_{1+(1 / 2)}=0
\end{gathered}
$$

$$
\begin{gathered}
\tilde{p}_{i+1}\left(\frac{1}{Z_{i+1}-Z_{i}}+\frac{1}{2} \frac{g \overline{\bar{\rho}}_{i+(1 / 2)}}{\overline{\bar{p}}_{i+(1 / 2)}}\right) \\
-\tilde{p}_{i}\left(\frac{1}{Z_{i+1}-Z_{i}}-\frac{1}{2} \frac{g \overline{\bar{\rho}}_{i+(1 / 2)}}{\overline{\bar{\rho}}_{i+(1 / 2)}}\right) \\
-\frac{R g \overline{\bar{\rho}}^{2}{ }_{i+(1 / 2)}}{\overline{\bar{p}}_{i+(1 / 2)} C_{p}} \tilde{S}_{i+(1 / 2)}=0 \\
(i=1,2,3,4)
\end{gathered}
$$

where the wavy symbol $(\sim)$ denotes the amplitude of wave disturbance which is a function of vertical layer number $i . k$ is the wave number and $C$ is a complex number $C_{R}+i C_{i}$, where $C_{R}$ is the phase velocity and $k C_{i}$ gives the growth rate of the wave disturbance. As mentioned before, we shall discuss only the stationary waves whose phase velocity are zero.

\subsection{Cloud model in the finite difference scheme}

We consider model clouds having their tops at three discrete levels. As three prediction levels of static energy are included in our model, three types of clouds are considered as shown in Fig. 1. The $H$-type is the highest type whose top reaches the $Z_{5}$-level $(10 \mathrm{~km})$, the $M$-type is the middle one whose top is at the $Z_{4}$-level $(7 \mathrm{~km})$ and the $L$-type is the lowest one whose top is at the $Z_{3}$-level $(4 \mathrm{~km})$.

Heating function $Q_{c}(Z)$ is written in the finite difference scheme as following

$$
\begin{aligned}
Q_{c}\left(Z_{i+(1 / 2)}\right)= & {\left[D ( Z _ { i + 1 } ) \left\{\hat{S}_{c}\left(\lambda_{n D-1}\right)-\overline{\bar{S}}\left(Z_{i_{+1}}\right)\right.\right.} \\
& \left.-L \hat{l}_{c}\left(\lambda_{n D-1}\right)\right\}+D\left(Z_{i}\right)\left\{\hat{S}_{c}\left(\lambda_{n D}\right)\right. \\
& \left.\left.-S\left(Z_{i}\right)-L \hat{l}_{c}\left(\lambda_{n D}\right)\right\}\right] / 2 \\
& +\left.\frac{\partial \bar{S}}{\partial Z}\right|_{i+(2 / 1)} M_{c}\left(Z_{i+(1 / 2)}\right)
\end{aligned}
$$

where subscript $n_{D}(=5-i+1)$ denotes the cloud type whose top is at the $Z_{i}$-level. Here the detrainment terms in Eq. (3.6) is expressed by the average of those at adjacent two levels where detrainment are defined.

For the evaluation of $\lambda, \hat{S}_{c}$ and $\hat{l}_{c}$ for each type, we use the following finite difference version of the continuity relation of mass and moist static energy $(h)$ for clouds. Original (differential) forms of these equations are found in Arakawa and Schubert (1974). According to them normalized cloud mass flux $\eta$ is determined by the following relations.

$$
\begin{aligned}
& \eta\left(Z_{i+(1 / 2)}, \lambda_{n}\right)= \eta\left(Z_{i_{-}(1 / 2)}, \lambda_{n}\right) \\
& \times\left(1+\lambda_{n}\left(Z_{i_{+}(1 / 2)}-Z_{\left.\left.i_{-}(1 / 2)\right)\right)}\right.\right. \\
&(i=2, \cdots, I D-1) \\
& \gamma\left(Z_{I D}, \lambda_{n}\right)=\eta\left(\lambda_{n}\right)=\eta\left(Z_{I D-(1 / 2)}, \lambda_{n}\right)
\end{aligned}
$$




$$
\begin{array}{r}
\times\left(1+\lambda_{n}\left(Z_{I D}-Z_{I D-(1 / 2)}\right)\right) \\
(i=I D)
\end{array}
$$

$$
\eta\left(Z_{i+(1 / 2)}, \lambda_{n}\right)=0 \quad(i>I D)
$$

and

$$
\begin{aligned}
& \eta\left(Z_{+(1 / 2)}, \lambda_{n}\right) h_{c}\left(Z_{+(1 / 2)}, \lambda_{n}\right) \\
& =\eta\left(Z_{i-(1 / 2)}, \lambda_{n}\right) h_{c}\left(Z_{i-(1 / 2)}, \lambda_{n}\right) \\
& +\overline{\bar{h}}\left(Z_{i}\right) \lambda_{n} \eta\left(Z_{i-(1 / 2)}, \lambda_{n}\right)\left(Z_{i+(1 / 2)}-Z_{-(1 / 2)}\right) \\
& \multicolumn{2}{c}{(i=2, \cdots, I D)} \\
& \eta\left(Z_{I D}, \lambda_{n}\right) h_{c}\left(Z_{I D}, \lambda_{n}\right)=\hat{\eta}\left(\lambda_{n}\right) \hat{h}_{c}\left(\lambda_{n}\right) \\
& =\eta\left(Z_{I D-(1 / 2)}, \lambda_{n}\right) h_{c}\left(Z_{I D-(1 / 2)}, \lambda_{n}\right) \\
& +\overline{\bar{h}}\left(Z_{I D}\right) \lambda_{n} \eta\left(Z_{I D-(1 / 2)}, \lambda_{n}\right)\left(Z_{I D-}-Z_{I D-(1 / 2)}\right) \\
& \quad(i=I D)
\end{aligned}
$$

Hereafter, $n=1$ corresponds to the $H$-type, $n=$ 2 to the $M$-type and $n=3$ to the $L$-type. Subscript $I D(=5-n+1)$ denotes the level number of detrainment for each $n$. While $\hat{\eta}$ and $\hat{h}_{c}$ are the value of $\eta(Z, \lambda)$ and $h_{c}(Z, \lambda)$ at the cloud top level for each $n$.

Following Arakawa and Schubert (1974), we adopt following two relations as the cloud base condition

$$
\begin{aligned}
& h_{c}\left(Z_{B}, \lambda_{n}\right)=h_{M} \\
& l_{c}\left(Z_{B}, \lambda_{n}\right)=l_{M}=0
\end{aligned}
$$

where subscript $M$ denotes value in the mixed layer. Further, it is assumed that $h_{M}$ must lie between $\overline{\bar{h}}{ }^{*}\left(Z_{B}\right)$ and $\overline{\bar{h}}\left(Z_{B}\right)$, as it is so in the ordinary tropical region.

$$
\overline{\bar{h}}\left(Z_{B}\right) \leqq h_{M} \leqq \overline{\bar{h}^{*}}\left(Z_{B}\right)
$$

On the other hand, following vanishing buoyancy condition must be satisfied at the cloud top level for all types

$$
\begin{gathered}
\hat{h}_{c}\left(\lambda_{n}\right)=\overline{\bar{h}}^{*}\left(Z_{I D}\right)-\left(1+\gamma\left(Z_{I D}\right)\right) L \varepsilon\left(Z_{I D}\right) \delta \\
/\left(1+\gamma\left(Z_{I D}\right) \varepsilon\left(Z_{I D}\right) \delta\right)\left(\overline{\bar{q}}^{*}\left(Z_{I D}\right)-\overline{\bar{q}}\left(Z_{I D}\right)\right)
\end{gathered}
$$

Using Newton's iteration technique we determine the entrainment rates and further $\eta$ and $h_{c}$ for each type under a given $h_{M}$ and a given basic field to satisfy the condition of Eq. (3.12). $S_{c}\left(Z, \lambda_{n}\right)$ and $q_{c}\left(Z, \lambda_{n}\right)$ are also determined by $h_{c}\left(Z, \lambda_{n}\right)$ thus obtained using following saturation condition. (cf. Arakawa et al. (1975))

$$
\begin{aligned}
& S_{c}\left(Z_{i+(1 / 2)}, \lambda_{n}\right)=\left(1 /\left(1+\gamma\left(Z_{+(1 / 2)}\right)\right)\right) \\
& \quad \times\left(h_{c}\left(Z_{i+(1 / 2)}, \lambda_{n}\right)-\overline{\bar{h}}^{*}\left(Z_{i+(1 / 2)}\right)\right) \\
& \quad+\overline{\bar{S}}\left(Z_{i+(1 / 2)}\right) \\
& \quad q_{c}\left(Z_{i+(1 / 2)}, \lambda_{n}\right)=\left(\gamma\left(Z_{i+(1 / 2)}\right) / L\left(1+\gamma\left(Z_{i+(1 / 2)}\right)\right)\right. \\
& \quad \times\left(h_{c}\left(Z_{i+(1 / 2)}, \lambda_{n}\right)-\overline{\bar{h}}^{*}\left(Z_{i+(12)}\right)\right) \\
& \quad+\overline{\bar{q}}^{*}\left(Z_{i+(1 / 2)}\right)
\end{aligned}
$$

In order to determine liquid water content in clouds the continuity relation of total water con- tent becomes necessary. The finite difference representation of total water content may be written by

$$
\begin{aligned}
\eta & \left(Z_{i+(1 / 2)}, \lambda_{n}\right)\left(q_{c}\left(Z_{i+(1 / 2)}, \lambda_{n}\right)\right. \\
\quad & \left.+l_{c}\left(Z_{i+(1 / 2)}, \lambda_{n}\right)\right)=\eta\left(Z_{i-(1 / 2)}, \lambda_{n}\right) \\
& \times\left(q_{c}\left(Z_{i-(1 / 2)}, \lambda_{n}\right)+l_{c}\left(Z_{i-(1 / 2)}, \lambda_{n}\right)\right) \\
& +\bar{q}\left(Z_{i}\right) \lambda_{n} \eta\left(Z_{i-(1 / 2)}, \lambda_{n}\right)\left(Z_{i+(1 / 2)}-Z_{i-(1 / 2)}\right) \\
& -\eta\left(Z_{i_{+}(1 / 2)}, \lambda_{n}\right) l_{c}\left(Z_{i_{+}((1 / 2)}, \lambda_{n}\right) \\
& \times\left(Z_{i+(1 / 2)}-Z_{i-(1 / 2)}\right) C_{0}
\end{aligned}
$$

if we use the same parameterization of the conversion from the cloud droplets to precipitation which was used by Arakawa and Schubert (1974). We adopt the same constant parameter, $C_{0}=$ 0.002 as chosen by them. Solving Eq. (3.14), we obtain $l_{c}\left(Z_{i}, \lambda_{n}\right)$ and $\hat{l}_{c}\left(\lambda_{n}\right)$.

$M_{c}(Z)$ and $D(Z)$ is Eq. (3.6) are expressed by

$$
\begin{aligned}
& \left(\begin{array}{l}
M_{c}\left(Z_{1+(1 / 2)}\right) \\
M_{c}\left(Z_{2+(1 / 2)}\right) \\
M_{c}\left(Z_{3+(1 / 2)}\right) \\
M_{c}\left(Z_{4+(1 / 2)}\right)
\end{array}\right) \\
& =\left(\begin{array}{ccc}
1 & 1 & 1 \\
\eta\left(Z_{2+(1 / 2)}, \lambda_{1}\right) & \eta\left(Z_{2+(1 / 2)}, \lambda_{2}\right) & \eta\left(Z_{2+(1 / 2)}, \lambda_{3}\right) \\
\eta\left(Z_{3+(1 / 2)}, \lambda_{1}\right) & \eta\left(Z_{3+(1 / 2)}, \lambda_{2}\right) & 0 \\
\eta\left(Z_{4+(1 / 2)}, \lambda_{1}\right) & 0 & 0
\end{array}\right) \\
& \times\left(\begin{array}{l}
m_{B}\left(\lambda_{1}\right) \\
m_{-}\left(\lambda_{2}\right) \\
m_{B}\left(\lambda_{3}\right)
\end{array}\right) \\
& M_{B}=M_{c}\left(Z_{1+(1 / 2)}\right)=m_{B}\left(\lambda_{1}\right)+m_{B}\left(\lambda_{2}\right)+m_{B}\left(\lambda_{3}\right) \\
& \left(\begin{array}{l}
D\left(Z_{3}\right) \\
D\left(Z_{4}\right) \\
D\left(Z_{5}\right)
\end{array}\right) \\
& =\left(\begin{array}{cccc}
0 & 0 & \hat{\eta}\left(\lambda_{3}\right) /\left(Z_{3+(1 / 2)}-Z_{2+(1 / 2)}\right) \\
0 & \hat{\eta}\left(\lambda_{2}\right) /\left(Z_{4+(1 / 2)}-Z_{3+(1 / 2)}\right) & 0 \\
\hat{\gamma}\left(\lambda_{1}\right) /\left(Z_{5+(1 / 2)}-Z_{4+(1 / 2)}\right) & 0 & 0
\end{array}\right) \\
& \times\left(\begin{array}{l}
m_{B}\left(\lambda_{1}\right) \\
m_{B}\left(\lambda_{2}\right) \\
m_{B}\left(\lambda_{3}\right)
\end{array}\right) \\
& D\left(Z_{1}\right)=D\left(Z_{2}\right)=0
\end{aligned}
$$

3.3 Formulation of $\partial A(\lambda) / \partial t$ in the finite difference scheme

The cloud work function of the $n$-type is defined in the finite difference scheme as follows by using the assumption mentioned in the section 2 .

$$
\begin{aligned}
A\left(\lambda_{n}\right)= & \sum_{i=1}^{I D-1} \eta\left(Z_{i_{+}(1 / 2)}, \lambda_{n}\right)\left\{\overline{\bar{\rho}}\left(Z_{+(1 / 2)}\right)\right. \\
& \times \beta\left(Z_{i_{+}(1 / 2)}\right)\left(h_{c}\left(Z_{\left.i_{+1} / 2\right)}, \lambda_{n}\right)\right. \\
& \left.-\bar{h}^{*}\left(Z_{i_{+}(1 / 2)}\right)\right)+g \delta\left(\bar{q}^{*}\left(Z_{i_{+}(1 / 2)}\right)\right.
\end{aligned}
$$


where

$$
\left.\left.-\bar{q}\left(Z_{i+(1 / 2)}\right)\right)\right\} \Delta Z_{i}
$$

$$
\begin{aligned}
& \Delta Z_{i}=Z_{i+1}-Z_{i}(i=2,3,4) \text { and } \\
& \Delta Z_{1}=\left(Z_{2}-Z_{1}\right) / 2
\end{aligned}
$$

The finite difference representation of $\partial A / \partial t$ is

$$
\begin{aligned}
\frac{\partial A\left(\lambda_{n}\right)}{\partial t}= & \sum_{i=1}^{I D-1} \eta\left(Z_{i+(1 / 2)}, \lambda_{n}\right) \overline{\bar{\rho}}\left(Z_{+(1 / 2)}\right) \\
& \times\left\{b\left(Z_{i+(1 / 2)}, \lambda_{n}\right) \lambda_{n} \frac{\partial \bar{h}\left(Z_{i+(1 / 2)}\right)}{\partial t}\right. \\
& \left.-\alpha\left(Z_{i+(1 / 2)}\right) \frac{\partial \overline{S_{v}}\left(Z_{i+(1 / 2)}\right)}{\partial t}\right\} \Delta Z_{i} \\
& -\overline{\bar{\rho}}\left(Z _ { 1 + ( 1 / 2 ) } \frac { \partial Z _ { B } } { \partial t } \left\{-\alpha\left(Z_{1+(1 / 2)}\right) \Delta S_{v}\right.\right. \\
& +\lambda_{n} \Delta h b\left(Z_{1+(1 / 2)}, \lambda_{n}\right) \\
& \left.-\lambda_{n} A\left(\lambda_{n}\right) / \overline{\bar{\rho}}\left(Z_{1+(1 / 2)}\right)\right\} \\
& (n=1,2,3)
\end{aligned}
$$

where

$$
\begin{aligned}
& \overline{\bar{\rho}}\left(Z_{i+(1 / 2)}\right) b\left(Z_{i+(1 / 2)}, \lambda_{n}\right) \\
= & \sum_{i^{\prime}=i}^{I D-1} \overline{\bar{\rho}}\left(Z_{i^{\prime}+(1 / 2)}\right) \beta\left(Z_{i^{\prime}+(1 / 2)}\right) \Delta Z_{i^{\prime}}
\end{aligned}
$$

The time derivative terms in Eq. (3.18) are written as

$$
\begin{aligned}
& \overline{\bar{\rho}}\left(Z_{i+(1 / 2)}\right) \frac{\partial \bar{S}_{v}\left(Z_{i+(1 / 2)}\right)}{\partial t}=-L(1-\varepsilon \delta) \\
& \quad \times\left\{D\left(Z_{i+1}\right) \hat{l}_{c}\left(\lambda_{5-i}\right)+D\left(Z_{i}\right) \hat{l}_{c}\left(\lambda_{5-i+1}\right)\right\} / 2 \\
& \quad+\frac{\partial \bar{S}_{v}}{\partial Z} \mid\left\{M_{c}\left(Z_{i+(1 / 2)}\right)\right. \\
& \left.\quad-\rho\left(Z_{i+(1 / 2)}\right) \bar{w}\left(Z_{i+(1 / 2)}\right)\right\} \\
& \rho\left(\left(Z_{i+(1 / 2)}\right) \frac{\partial \bar{h}\left(Z_{i+(1 / 2)}\right)}{\partial t}\right. \\
& =\left\{D ( Z _ { i + 1 } ) \left[\hat{h}_{c}\left(\lambda_{5-i}\right)-\bar{h}^{*}\left(Z_{i+1}\right)\right.\right. \\
& \left.\quad+L\left(\overline{\bar{q}}^{*}\left(Z_{i+1}\right)-\overline{\bar{q}}\left(Z_{i+1}\right)\right)\right] \\
& \quad+D\left(Z_{i}\right)\left[\hat{h}_{c}\left(\lambda_{5-i+1}\right)-\bar{h}^{*}\left(Z_{i}\right)\right. \\
& \left.\left.\quad+L\left(\overline{\bar{q}}^{*}\left(Z_{i}\right)-\overline{\bar{q}}\left(Z_{i}\right)\right)\right]\right\} / 2 \\
& \quad+\frac{\partial \overline{\bar{h}}}{\partial Z} \mid\left\{M_{c}\left(Z_{i+(1 / 2)}\right)\right. \\
& \left.\quad-\overline{i+(1 / 2)}\left(Z_{i+(1 / 2)}\right) \bar{w}\left(Z_{i+(1 / 2)}\right)\right\} \\
& \frac{\partial Z_{B}}{\partial t}=\left(1 / \overline{\bar{\rho}}\left(Z_{1+(1 / 2)}\right)\right)\left(-M_{c}\left(Z_{1+(1 / 2)}\right)\right. \\
& \left.\quad+\overline{\bar{\rho}}\left(Z_{1+(1 / 2)}\right) \bar{w}\left(Z_{1+(1 / 2)}\right)\right)
\end{aligned}
$$

Substituting Eq. (3.19)-(3.21) into Eq. (3.18) and rewriting $M_{c}$ and $D$ by $m_{B}(\lambda)$, we obtain the following matrix formula.

$$
\left(\begin{array}{l}
\frac{\partial A\left(\lambda_{1}\right)}{\partial t} \\
\frac{\partial A\left(\lambda_{2}\right)}{\partial t} \\
\frac{\partial A\left(\lambda_{3}\right)}{\partial t}
\end{array}\right)=\left(\begin{array}{ccc}
K_{D 11} & K_{D 12} & K_{D 13} \\
0 & K_{D 22} & K_{D 23} \\
0 & 0 & K_{D 33}
\end{array}\right)\left(\begin{array}{l}
m_{B}\left(\lambda_{1}\right) \\
m_{B}\left(\lambda_{2}\right) \\
m_{b}\left(\lambda_{3}\right)
\end{array}\right)
$$

$$
\begin{aligned}
& +\left(\begin{array}{lll}
K_{V 11} & K_{V 12} & K_{V 13} \\
K_{V 21} & K_{V 22} & K_{V 23} \\
K_{V 31} & K_{V 32} & K_{V 33}
\end{array}\right)\left(\begin{array}{l}
m_{B}\left(\lambda_{1}\right) \\
m_{B}\left(\lambda_{2}\right) \\
m_{B}\left(\lambda_{3}\right)
\end{array}\right) \\
& +\left(\begin{array}{lll}
K_{\mathrm{MIX} 1} & K_{\mathrm{MIX} 1} & K_{\mathrm{MIX} 1} \\
K_{\mathrm{MIX} 2} & K_{\mathrm{MIX} 2} & K_{\mathrm{MIX} 2} \\
K_{\mathrm{MIX} 3} & K_{\mathrm{MIX} 3} & K_{\mathrm{MIX} 3}
\end{array}\right)\left(\begin{array}{l}
m_{B}\left(\lambda_{1}\right) \\
m_{B}\left(\lambda_{2}\right) \\
m_{b}\left(\lambda_{3}\right)
\end{array}\right) \\
& -\left(\begin{array}{llll}
F_{c 11} & F_{c 21} & F_{c 31} & F_{c 41} \\
F_{c 12} & F_{c 22} & F_{c 32} & F_{c 42} \\
F_{c 13} & F_{c 23} & F_{c 33} & F_{c 43}
\end{array}\right) \\
& \times\left(\begin{array}{l}
\overline{\bar{\rho}}_{1} \bar{w}_{+(1 / 2)} \\
\overline{\bar{\rho}} \bar{w}_{2+(1 / 2)} \\
\overline{\bar{\rho}} \bar{w}_{3+(1 / 2)} \\
\overline{\bar{\rho}} \bar{w}_{4+(1 / 2)}
\end{array}\right)--\left(\begin{array}{l}
F_{M 1} \\
F_{M 2} \\
F_{M 3}
\end{array}\right) \bar{\rho} \bar{w}_{1+(1 / 2)}
\end{aligned}
$$

Formulas for the elements of matrices $\left\{K_{D}\right\}$, $\left\{K_{v}\right\},\left\{K_{M I X}\right\},\left\{F_{c}\right\}$ and $\left\{F_{M}\right\}$ are given in appendix B.

\subsection{The determination of $m_{B}(\lambda)$}

Because three types are considered in the present model as shown in Fig. 1, seven combinations, (HML), (HM), (HL), (ML), (H), (M) and (L) are possible. Here, each letter in parenthesis denotes an existing cloud type.

Let a combination be assumed. For all of the existing types included in the combination, $\partial A\left(\lambda_{\mathrm{EXT}}\right) / \partial t$ should vanish and for all of the suppressed type, $m_{B}(\lambda)$ should be zero by assumption. Thus,

$$
\begin{aligned}
\left\{\frac{\partial A\left(\lambda_{\mathrm{EXT}}\right)}{\partial t}\right\}= & \left\{K\left(\lambda_{\mathrm{EXT}} \lambda^{\prime}{ }_{\mathrm{EXT}}\right)\right\}\left\{m_{E}\left(\lambda_{\mathrm{EXT}}^{\prime}\right)\right\} \\
& -\left\{F\left(\lambda_{\mathrm{EXT}}\right)\right\}\{\overline{\bar{\mu}} \bar{w}\}=0
\end{aligned}
$$

where subscript (EXT) denotes an existing type. Note that the above equation applies only to existing type clouds, and we have equations as many as the number of unknown, $m_{B}\left(\lambda_{\mathrm{EXT}}\right)$. The first term of Eq. (3.23) expresses the decreasing effect of $A\left(\lambda_{\mathrm{EXT}}\right)$ by all of the existing type included in the combination, while the second the increasing effect of it by $\overline{\bar{\rho}} \bar{w}$.

Thus, each $m_{B}\left(\lambda_{\mathrm{EXT}}\right)$ of the existing type is expressed as a linear function of $\overline{\bar{\rho}} \bar{w}$.

$$
\begin{aligned}
\left\{m_{B}\left(\lambda_{\mathrm{EXT}}\right)\right\}= & \left\{K\left(\lambda_{\mathrm{EXT}}, \lambda_{\mathrm{EXT}}^{\prime}\right)\right\}^{-1} \\
& \times\left\{F\left(\lambda_{\mathrm{EXT}}\right)\right\}\{\overline{\bar{\rho}} \bar{w}\}
\end{aligned}
$$

where $\{K\}^{-1}$ is the inverse matrix of $\{K\}$. By using Eq. (3.15) and (3.16), $Q_{c}\left(Z_{i}+1 / 2\right)$ is expressed as a linear function of $\overline{\bar{\rho}} \bar{w}$ at all levels for the combination.

Then, basic equation system from (3.1) to (3.5) which is a linear homogenous equation system, can be solved as an eigen value problem. A 
growth rate is obtained as an eigen value and a set of amplitude function such as $\bar{u}, \bar{v}, \bar{w}, \bar{s}, \bar{p}$, as the corresponding eigen solution. Here, the over-bar denotes amplitude function of stationary waves. $m_{B}(\lambda)$ of the existing type are estimated by substituting $\overline{\bar{\rho}} \bar{w}$ into Eq. (3.24). By using $\overline{\bar{\rho}} \bar{w}$ and $m_{B}\left(\lambda_{\mathrm{EXT}}\right)$ thus obtained $\partial A\left(\lambda_{\mathrm{sup}}\right) / \partial t$ is estimated for each of the suppressed type by using following equation,

$$
\begin{aligned}
\left\{\frac{\partial A\left(\lambda_{\text {sup }}\right)}{\partial t}\right\} & =\left\{K\left(\lambda_{\text {sup }}, \lambda^{\prime}{ }_{\mathrm{EXT}}\right)\right\}\left\{m_{B}\left(\lambda_{\mathrm{EXT}}^{\prime}\right)\right\} \\
& -\left\{F\left(\lambda_{\text {sup }}\right)\right\}\{\overline{\bar{\rho}} \bar{w}\}
\end{aligned}
$$

where subscript (SUP) denotes a suppressed type. The first term is the decreasing effect of $A\left(\lambda_{\text {sup }}\right)$ by the existing types included in the combination, while the second is increasing effect of it by $\rho w$.

For a set of eigen solution that satisfies the quasi equilibrium assumption, $m_{B}\left(\lambda_{\mathrm{EXT}}\right)$ must have positive value for each of the existing type (the upper condition) and $A\left(\lambda_{\text {sup }}\right)$ must decrease with time for each of the suppressed type (the lower condition). As we do not know what combination produces such solution, we solve seven sets of eigen value problems for seven combinations. And then, we chose the solution that satisfies the condition (2.15).

In the present finite difference scheme, twelve eigen values are included for a cloud combination. In general, one or two stationary waves and eight propergating waves exist among these twelve, while the rest are trivial solutions whose $C_{R}$ and $C_{i}$ are zero. In the present analysis, we will deal with only stationary waves.

\section{Characteristic features of seven modes}

The purpose of this section is to examine the combination which produces the solution satisfying the quasi-equilibrium assumption is uniquely determined or there are many solutions under a given basic field and a cloud base condition. Further, generally there is a possibility that no physically valid solution exist for any combination.

The uniqueness of solution in this paper, does not mean that the numerical solution of Fredholm integral equation of the first kind for $\partial A(\lambda) / \partial t$ is always uniquely determined (cf. Arakawa and Schubert (1974)) but that the cloud combination, in other words, the mode which satisfies the quasiequilibrium assumption, is only one for all wavelength under a given basic field and cloud base condition.

The growth rates of seven modes are shown in
Fig. $2 b$ as function of disturbances' wavelength for a typical example. The basic field and the cloud base condition adopted in this example, are shown in Fig. 2a. The names of modes correspond to the combination of existing cloud types assumed. The type whose $m_{B}(\lambda)$ becomes negative is underlined in parenthesis. The signs of $\partial A(\lambda) /$ $\partial t$ of the three clouds are shown in brackets; the upper one is $\partial A\left(\lambda_{H}\right) / \partial t$, the middle one is $\partial A\left(\lambda_{M}\right) / \partial t$ and the lowest one is $\partial A\left(\lambda_{L}\right) / \partial t$. As for the existing type, $\partial A(\lambda) / \partial t$ is, of course, zero by assumption. Combining these information we can classify the results into three groups, distinguished by three lines. The solid lines show the modes that satisfy the quasi-equilibrium assumption and hence acceptable solution. The chain lines show modes in which. some negative $m_{B}\left(\lambda_{\mathrm{EXT}}\right)$ are included. On the other hand, dashed lines show the mode in which some $A\left(\lambda_{\text {sup }}\right)$ increase with time. Note that the mode satisfying the quasi-equilibrium assumption is only one for all wavelength. Thus the uniqueness of solution is realized in this example. In general, only one valid solution exist for almost all basic fields although there are some exceptional cases described in the later section. The mode shown by chain lines means that too many cloud types are included in that mode in order to cancel the increase of $A\left(\lambda_{\mathrm{ExT}}\right)$ caused by the large scale upward motion. Thus the modes may be considered as the cases of over-stabilization by clouds. On the contrary, the modes shown by dashed lines mean that $A\left(\lambda_{\text {sup }}\right)$ increases because the effects to decrease it by others of the existing type are

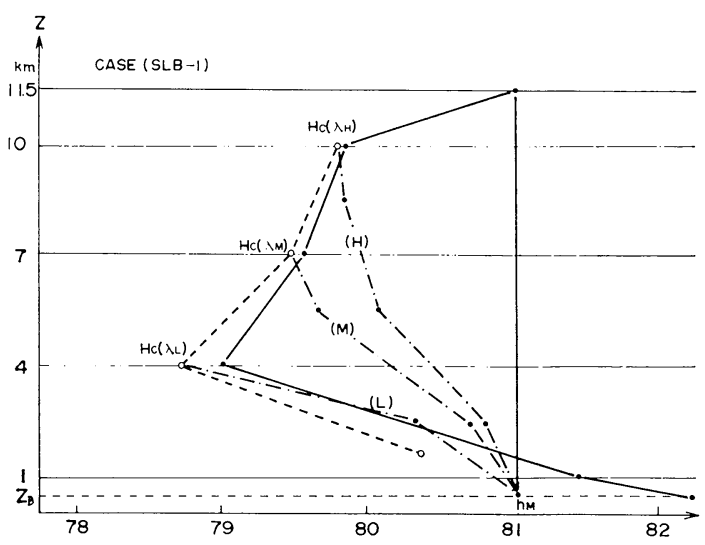

Fig. 2a Basic field for case (SLB-1). Solid lines show saturation moist static energy in cloud environment and chain lines those in cloud for three types. Dashed line shows $h_{c}(\lambda)$. 


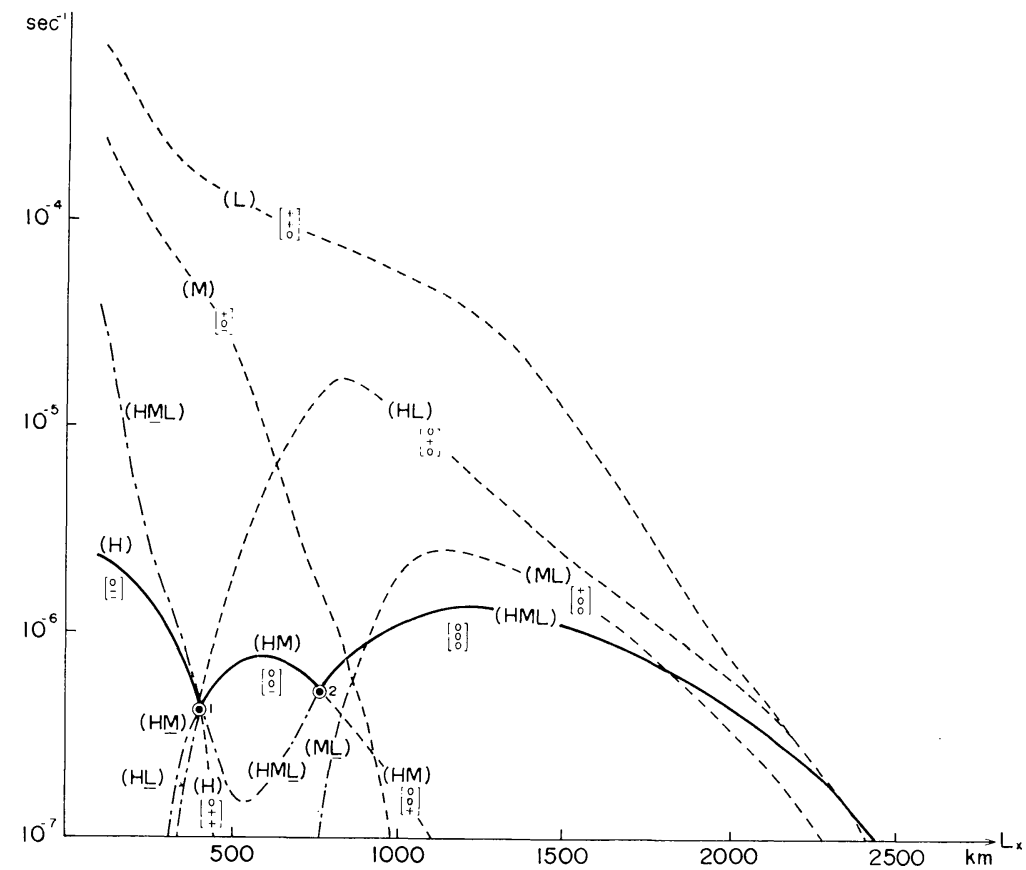

Fig. 2b Growth rates of seven modes for case (SLB-1) are shown as functions of wavelength. For details see the text.

insufficient to cancel the increasing by large scale upward motion. Then the modes represent the cases of under-stabilization by clouds.

As shown in Fig. $2 b$, for shorter wavelengths where mode $(\mathrm{H})$ is only one solution, other modes including lower types besides $\mathrm{H}$-type, such as mode (HML), (HM) etc. become over-stabilized, while those including single type such as mode (M) and mode (L) become under-stabilized. This suggests that M-type and L-type are not needed at such short wavelength to satisfy the quasiequilibrium assumption because the increase of $A\left(\lambda_{M}\right)$ and $A\left(\lambda_{L}\right)$ by large scale motion is cancelled by the decreasing of cumulus-included subsidence from the $\mathrm{H}$-type. However, if $\mathrm{H}$-type does not exist, the decreasing of $A\left(\lambda_{H}\right)$ by $\mathrm{M}-$ and L-type is not sufficient to balance the increasing of it by large scale upward motion. In the range of longer wavelength where mode (HML) is only a valid solution, under-stabilization occurs, if any one of the suppressed type is included. In this range, the increasing of $A\left(\lambda_{\text {sup }}\right)$ by large scale upward motion is not cancelled by others of the existing type.

Further, we find in Fig. $2 b$ that the mode including lower type clouds appears as a valid solution with increasing wavelength. Namely, mode (H), mode (HM) and (HML) appear successively. For modes (HM) and (HML), short wave cut-off occurs by over-stabilization at the wavelength shown by $O_{1}$ and $O_{2}$ respectively, while for modes $(\mathrm{H})$ and $(\mathrm{HM})$, long wave cut-off occurs by under-stabilization. Fig. $2 b$ shows three maximum and two minimum points of growth rates and three modes seem to be discrete one another. However, this may be an apparent feature, which is due to the crudeness of the present finite difference scheme where only three cloud types are defined. Growth rate curve becomes more smooth in the model in which more cloud types are included as shown in next paper.

As for the present analysis, there are not cases where no solution exist for all seven combinations.

In the present section, we cite cumulus-included subsidence and large scale upward motion as effects which cause the time change of cloud work function. The effects of detrainment and time change of top level of the mixed layer, are also included together with them. However, the later two effects are much smaller than the former two quantitatively as described in the later section.

\section{Stability properties of unstable waves}

The convective heating function in the present model is dependent on thermodynamical quantities of a basic field such as $\overline{\bar{h}}_{i}, \overline{\bar{S}}_{v i}, \partial \overline{\bar{h} i} / \partial Z \delta \overline{\overline{S v}} / \delta Z$ $(i=1,2,3,4,5)$ and $h_{M}$. We shall investigate 
the stability properties of waves in several basic fields of different vertical distributions of temperature and water vapour.

Among three cloud kernels mentioned in section 3 , vertical mass flux kernel $\left[K_{v}\right]$ is larger than detrainment, $\left[K_{D}\right]$, and mixed layer kernels, [ $\left.K_{\mathrm{MIX}}\right]$, by more than one order in magnitude. This implies that the most important effect of cumulus clouds is due to the warming and drying by cumulus-induced subsidence. Thus growth rates may be sensitively dependent on the static stability and vertical distribution of water vapour. The cloud base condition $h_{M}$ has, of course, a significant effect on $Q_{c}$.

We shall consider effects of the static stability of a basic field in the lower layer $(1000 \mathrm{~m}-$ $4000 \mathrm{~m})$, the middle layer $(4000 \mathrm{~m}-7000 \mathrm{~m})$ and the upper layer $(7000 \mathrm{~m}-10000 \mathrm{~m})$ in the subsection (5.1). We shall consider the effects of vertical distribution of water vapour in the subsection (5.2). Finally, effects of $h_{M}$ are investigated in the sub-section (5.3).

In calculation in this study, the moist static

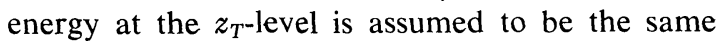
as $h_{M}$ to eliminate the possibility of clouds rising higher than the top level of the model atmosphere.

5.1 Stability properties of waves in several different stratifications

In the present sub-section, we shall describe some characteristic features of growth rate curves which are obtained under several different conditions of stratification. For this purpose, we use the following four cases of basic field such as case (SLA), case (SLB), case (SM) and case (SH). Fig. $3 \mathrm{a}, 4 \mathrm{a}, 5 \mathrm{a}$ and $6 \mathrm{a}$ show a series of vertical profile of temperature by saturation moist static energy $\left(\bar{h}^{*}\right)$ for these four cases. The temperature profile with letter $(\mathrm{J})$ approximately corresponds to the mean tropical atmosphere for hurricane season presented by Jordan (1958); temperature $Z_{1+1 / 2^{-}}, \quad Z_{2^{-}}, \quad Z_{3^{-}}, Z_{4^{-}}$and $Z_{5^{-}}$level are $22^{\circ} \mathrm{C}$, $19^{\circ} \mathrm{C}, 3^{\circ} \mathrm{C},-14^{\circ} \mathrm{C}$ and $-37^{\circ} \mathrm{C}$ respectively, and relative humidity at those levels are $80 \%, 80 \%$, $50 \%, 40 \%$, and $20 \%$ respectively. For the four cases, temperature in the lowest layer $(0-1000 \mathrm{~m})$ is fixed to the same value as that in case $(\mathrm{J})$, while those at $Z_{3^{-}}, Z_{4^{-}}$, and $Z_{5^{-}}$level are altered systematically for each case. On the other hand, vertical profile of relative humidity used in the present subsection is always the same as that in case $(\mathrm{J})$.

At first, we shall investigate effects of the static stability in the lower layer by using case (SLA) and (SLB). For case (SLA), we reduce the tem- perature at the $Z_{3}$-level gradually to decrease the static stability $(\partial \overline{\bar{S}} / \partial Z)$ in the lower layer, while the temperature at the $Z_{5}$-level is fixed to a constant. Therefore, as the lower layer becomes unstable, the upper layer becomes stable from case (SLA-1) to (SLA-7). The entrainment rates of three types are shown in table (1). As reducing temperature at the $Z_{3^{-}}$and $Z_{4}$-level, the entrainment rates of the M-type and the L-type clouds are increased, while that of the H-type is slightly decreased because of cooling in the middle and the lower layer under the assumption of a fixed height for each cloud type.

Fig. 3b shows the growth rates of unstable waves as functions of wavelength from cases (SLA-1) to (SLA-7). The cloud base condition for moist static energy $h_{M}$ is $81.02 \mathrm{cal} \mathrm{gm}^{-1}$ in these cases. It is seen in Fig. $3 b$ that three modes $(H)$, (HM) and (HML) appear successively as wavelength increases, in cases (J), (SLA-1), (SLA-2) and (SLA-3). When the lower layer is not so much unstable as in cases (J) and (SLA-1), mode (HML) is most dominant among three and wavelength of maximum growth rate is around $1000 \mathrm{~km}$, although the growth rates are less than $10^{-6} \mathrm{sec}^{-1}$. However, as the static stability of the lower layer becomes more unstable, the growth rates of mode (HM) considerably increase for short wavelength. For cases of strongly unstable lower layer, namely for cases (SLA-2), (SLA-3) and (SLA-4), mode (HM) becomes most unstable. Note that the wavelength of the maximum growth rate of mode (HM) shown by $\bigcirc$ becomes shorter and growth rate increases as the lower layer becomes more unstable. In case (SLA1) the most preferred scale of mode $(\mathrm{HM})$ is at

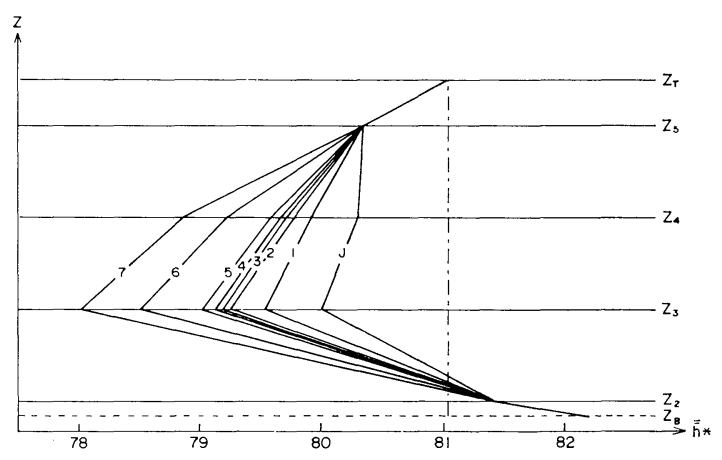

Fig. 3a Basic field for case (SLA-n) where $n$ denotes case number. Solid-lines show saturation moist static energy of cloud environment for each number. 


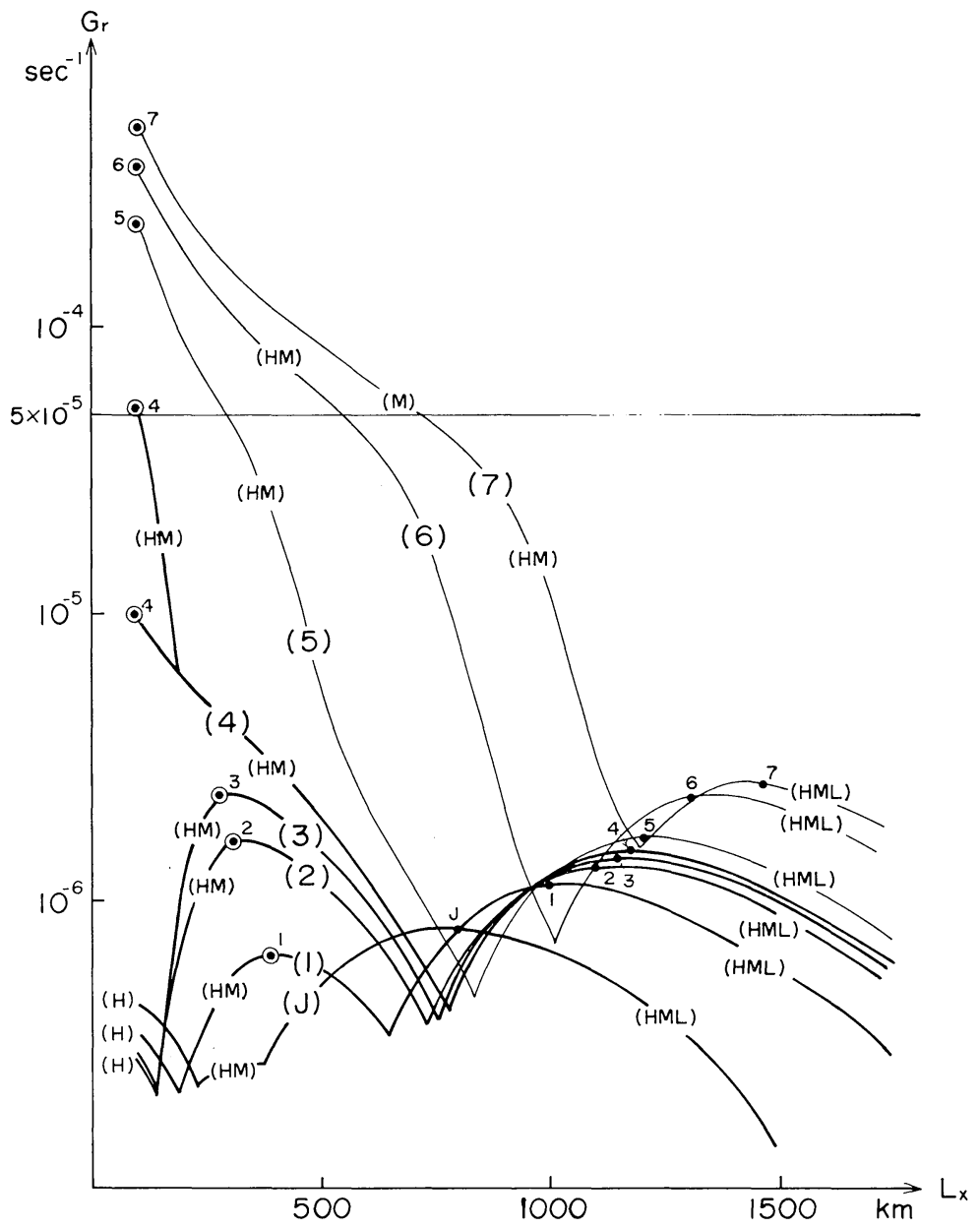

Fig. $3 b$ Growth rate of solution for case (SLA-n).

Table 1. entrainment rates of three cloud types for case (SLA) $(\% / \mathrm{km})$

\begin{tabular}{ccrr}
\hline CASE & $\begin{array}{c}\lambda_{H} \\
\times 10^{-5}\end{array}$ & $\begin{array}{c}\lambda_{M} \\
\times 10^{-5}\end{array}$ & $\begin{array}{r}\lambda_{L} \\
\times 10^{-5}\end{array}$ \\
\hline J & 4.80 & 7.14 & 27.70 \\
SLA-1 & 4.17 & 10.19 & 42.25 \\
SLA-2 & 3.93 & 11.80 & 50.79 \\
SLA-3 & 3.87 & 12.13 & 52.58 \\
SLA-4 & 3.83 & 12.45 & 54.38 \\
SLA-5 & 3.70 & 13.42 & 59.96 \\
SLA-6 & 3.31 & 16.69 & 80.23 \\
SLA-7 & 3.02 & 19.75 & 101.10 \\
\hline
\end{tabular}

$500 \mathrm{~km}$ or so, while in case (SLA-3) that is at about $300 \mathrm{~km}$. As increasing of growth rate of mode $(\mathrm{HM})$, mode $(\mathrm{H})$ tends to disappear.

When the lower layer is extremely unstable as in cases (SLA-4), (SLA-5), (SLA-6) and (SLA-7), mode (HM) occupies whole range of short wave- length. In these cases the most preferred scale occurs at the shortest wavelength and the short wave cut-off does not appear. However the growth rates of the mode exceed the Coriolis parameter $\left(5 \times 10^{-5} \mathrm{sec}^{-1}\right)$ for wavelength shorter than about $200 \mathrm{~km}$. An typical feature of structures of these waves is that inflow increases with height linearly at least in the lower layer. In this study, the waves whose growth rate exceeds Coriolis parameter at the shortest wavelength, are referred to as unstable stationary gravity waves, because for those waves quasi-geostrophic balance can not hold at all. Growth rates of these waves are shown by thin solid lines. In case (SLA-4) which may be considered as a transient case between the quasi-geostrophic mode and the gravity mode, two branches of mode (HM) appear for shorter wavelength. The uniqueness of solution is not realized. 
In order to investigate further the effect of the static stability of the lower layer, we consider a series of case (SLB). In this series, only the static stability in the lower layer is decreased, while those in the upper and the middle layer are fixed to the same values as those in case $(\mathrm{J})$ by reducing

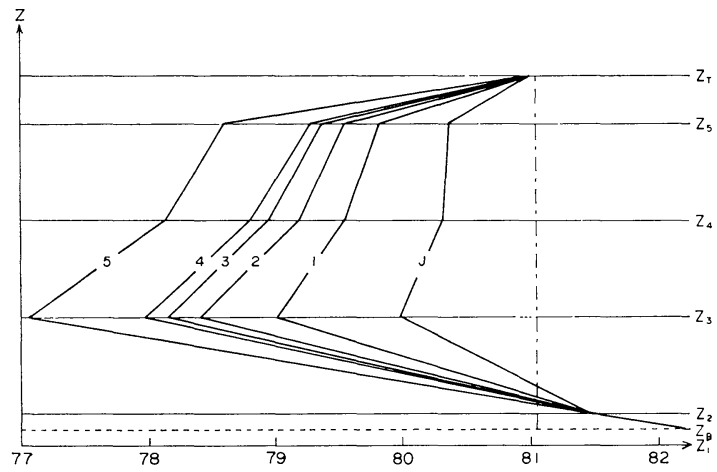

Fig. 4a Same as Fig. 3a except for case (SLB-n).
Table 2. same as table 1 except for case (SLB)

\begin{tabular}{crrr} 
CASE & $\begin{array}{c}\lambda_{H} \\
\times 10^{-5}\end{array}$ & $\begin{array}{c}\lambda_{M} \\
\times 10^{-5}\end{array}$ & \multicolumn{1}{c}{$\begin{array}{c}\lambda_{L} \\
\times 10^{-5}\end{array}$} \\
\hline J & 4.80 & 7.14 & 27.70 \\
SLB-1 & 7.97 & 13.42 & 59.97 \\
SLB-2 & 9.39 & 16.60 & 80.28 \\
SLB-3 & 10.12 & 18.82 & 94.92 \\
SLB-4 & 10.51 & 19.75 & 101.10 \\
SLB-5 & 13.00 & 25.99 & 139.89 \\
\hline
\end{tabular}

the temperature at all levels between $Z_{3}$ and $Z_{5}$. As a result entrainment rates of all three types are increased from case (SLB-1) to case (SLB-5). (cf. table (2))

Fig. 4b shows the growth rates for case (SLB) obtained by using the same value of $h_{M}$ as that of case (SLA). Fig. 4b also indicates that the growth rates for short wavelength increase as the lower layer becomes unstable. However, for this

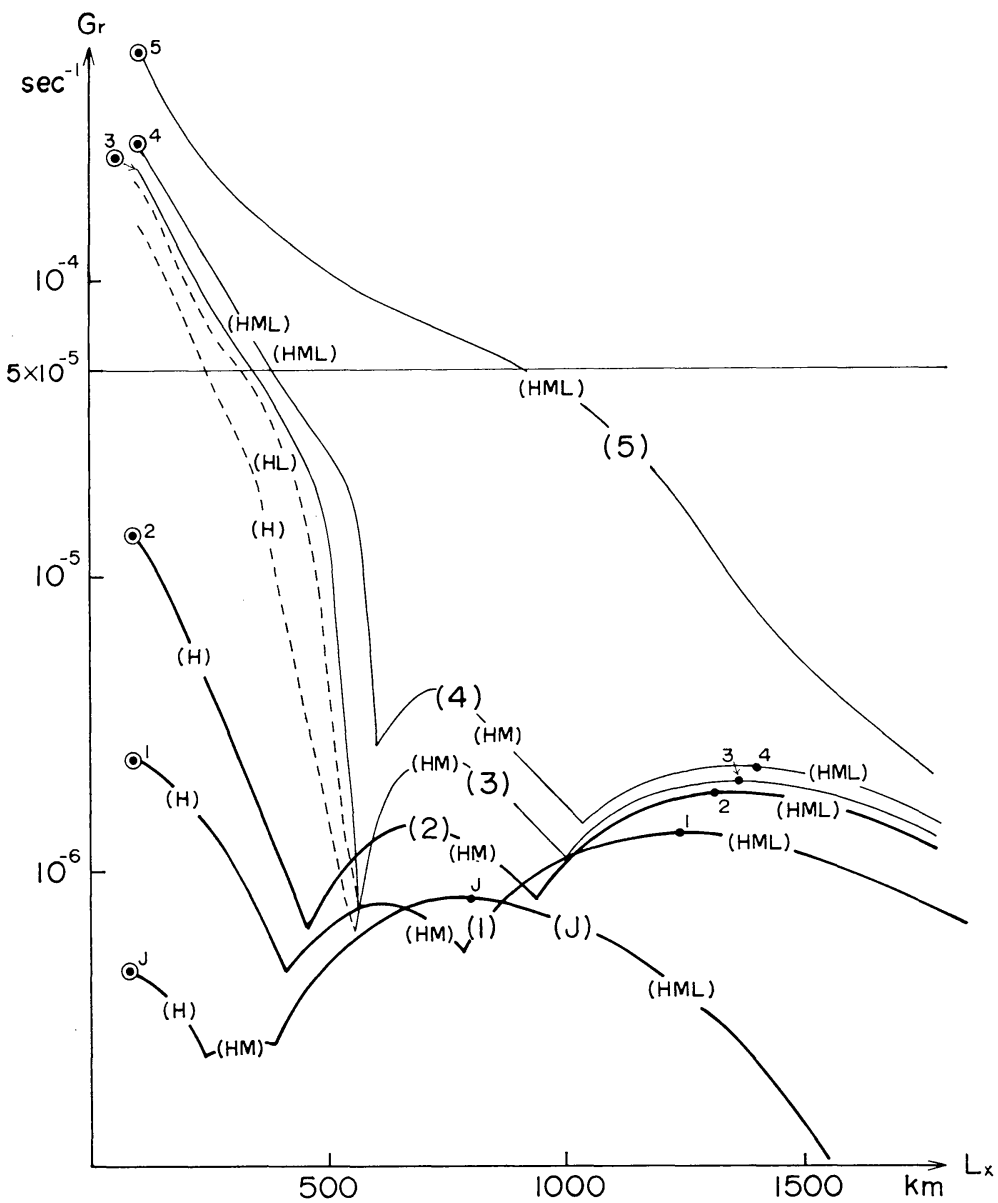

Fig. $4 b$ Same as Fig. 3b except for case (SLB-n). 
case the mode $(\mathrm{H})$ becomes most unstable, while mode (HM) does not increase so much as the mode $(\mathrm{H})$. The most preferred scale of mode $(\mathrm{H})$ is always at the shortest wavelength. When the lower layer becomes extremely unstable like case (SLB-3), (SLB-4) and (SLB-5), stationary gravity wave of mode (HML) appears for short wavelength. For the transient case of the case (SLB-3), the three modes (H), (HL) and (HML) become acceptable as solutions. Thus for case (SLB) the uniqueness also is not realized in the transient case.

Next, we shall consider the case (SM) to in-

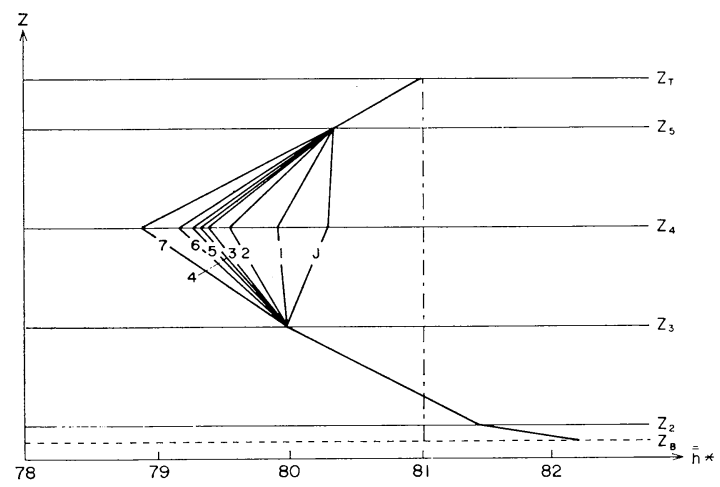

Fig. 5a Same as Fig. 3a except for case (SM-n).

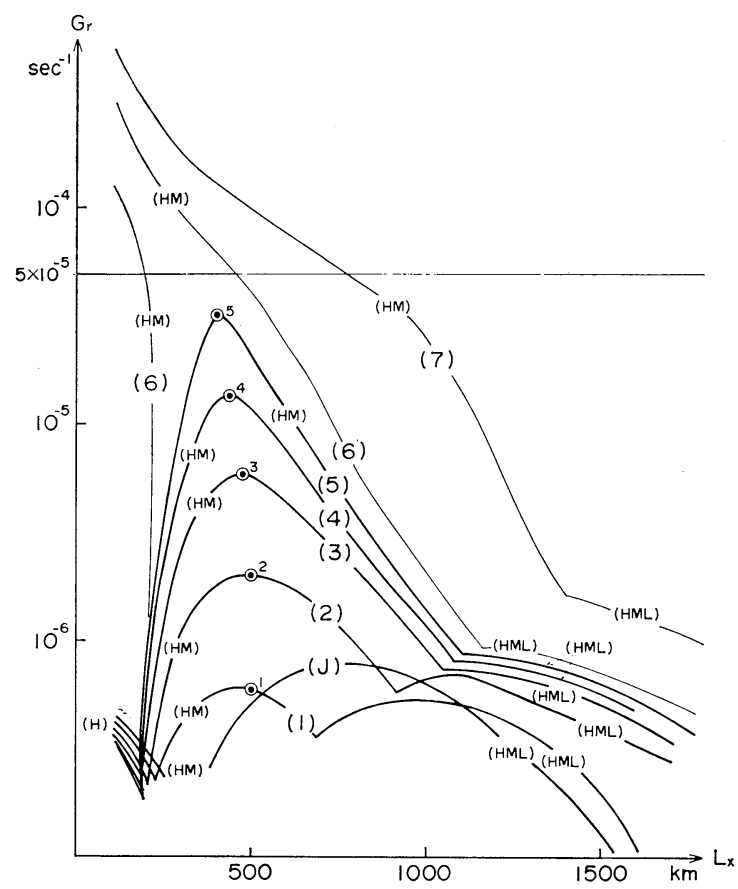

Fig. 5b Same as Fig. $3 b$ except for case (SM-n).
Table 3. samd as tablp 1 except for case (SM)

\begin{tabular}{cccc}
\hline CASE & $\begin{array}{c}\lambda_{I} \\
\times 10^{-5}\end{array}$ & $\begin{array}{c}\lambda_{M} \\
\times 10^{-5}\end{array}$ & $\begin{array}{c}\lambda_{L} \\
\times 10^{-5}\end{array}$ \\
\hline J & 4.80 & 7.14 & 27.70 \\
SM-1 & 4.49 & 11.39 & 27.70 \\
SM-2 & 4.22 & 16.93 & 27.70 \\
SM-3 & 4.10 & 20.20 & 27.70 \\
SM-4 & 4.05 & 21.64 & 27.70 \\
SM-5 & 4.04 & 22.38 & 27.70 \\
SM-6 & 3.98 & 23.92 & 27.70 \\
SM-7 & 3.78 & 32.95 & 27.70
\end{tabular}

vestigate the effect of the static stability in the middle layer by reducing the temperature at the $Z_{4}$-level. Temperature at all levels except the $Z_{4}$-level are the same as those of case $(\mathrm{J})$. Thus the static stability in the lower layer does not change in this case. However the stratification in the upper layer becomes stable as the middle layer becomes unstable. For case (SM), we use the same value of $h_{M}$ as those used for case (SLA) and (SLB). Table (3) shows the entrainment rates of three types for case (SM). The growth rates for case (SM) are shown in Fig. 5b. In this case, mode (HM) is most unstable and the most preferred scale is about $500 \mathrm{~km}$. As the static stability of the middle layer is reduced, the growth rates of mode (HM) increase considerably from case (SM-1) to (SM-4), but those of mode (H) tend to disappear. This situation is same as case (SLA) shown in Fig. 3b. For case (SM), the short wave cut off is very clear. It is observed that in this case, in the same manner as in the case (SLA) the most preferred scale of mode (HM) becomes smaller and the growth rate increases as the middle layer becomes more unstable. For the strongly unstable state, i.e., in the cases (SM-5) and (SM-6), the stationary gravity waves appear and in the transient case (SM-4), the uniqueness of solution is lost.

Finally, we investigate only the effect of the static stability of the upper layer. The effects of the increasing of static stability in the upper layer is included in case (SLA) and case (SM) simultaneously with decreasing of static stability in the middle and the lower layer. For case (SH), only the temperature at the $Z_{5}$-level is increased from case (SH-0) to case (SH-3), while those at other levels are kept the same as those of case (J). For case $(\mathrm{SH}), 81.53 \mathrm{cal} \cdot \mathrm{gm}^{-1}$ is adopted as $h_{M}$. Case $(\mathrm{J})^{\prime}$ is the same as case (J) except that the value of $h_{M}$ is $81.02 \mathrm{cal} \cdot \mathrm{gm}^{-1}$ for the later case. The 


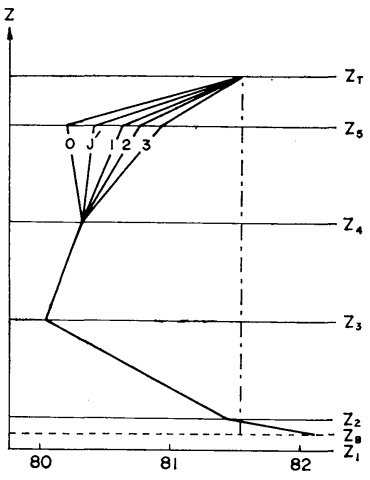

Fig. 6a Same as Fig. 3a except for case (SH-n).

entrainment rates of three types are shown in Table (4). The growth rate curves are shown in Fig. 6b. The most notable feature is that the growth rate of mode $(\mathrm{H})$ is much reduced as the static stability in the upper layer increases. On the contrary to mode $(\mathrm{H})$, the growth rate of mode (HM) increases considerably by increasing of the static stability in the upper layer.

As a conclusion of the present subsection, it

Table 4. same as table 1 except for case (SH)

\begin{tabular}{lccc}
\hline CASE & $\begin{array}{c}\lambda_{H} \\
\times 10^{-5}\end{array}$ & $\begin{array}{c}\lambda_{M} \\
\times 10^{-5}\end{array}$ & $\begin{array}{c}\lambda_{L} \\
\times 10^{-5}\end{array}$ \\
\hline SH-0 & 9.16 & 10.58 & 34.61 \\
$\mathbf{J}^{\prime}$ & 7.77 & 10.58 & 34.61 \\
SH-1 & 5.40 & 10.58 & 34.61 \\
SH-2 & 4.39 & 10.58 & 34.61 \\
SH-3 & 3.45 & 10.58 & 34.61 \\
\hline
\end{tabular}

may be pointed out that the typhoon-scale disturbance whose preferred scale is less than a few hundred kilometers becomes dominant as the lower part of atmosphere, the lower and the middle layers of the present model, becomes unstable. The disturbance belongs to either mode (H) or mode (HM). The mode which is selected is dependent on the static stability of upper layer. When the upper layer is stable, mode (HM) whose preferred scale is about $500 \mathrm{~km}$ tends to dominate. However when the stratification of the upper layer is fairly unstable, mode $(\mathrm{H})$ whose preferred scale is always at the shortest wavelength, becomes most unstable.

5.2 The effects of vertical distribution of water vapour

The effects of the vertical distribution of water vapour are partially included in the results described in the previous subsection with those of temperature. However, in order to clarify the effects independently, we investigate the properties of unstable waves produced in several basic fields with different distribution of water vapour using the same temperature distributions as case (J). Water vapour is usually concentrated in the lowest part of the atmosphere. Then we alter the values of relative humidities at the $Z_{1+(1 / 2)}$ level (the lowest layer) and at the $Z_{3}$-level while those at the $Z_{4^{-}}$and the $Z_{5}$-level are fixed to $40 \%$, and $0 \%$ respectively. In the following calculation, we adopt $81.53 \mathrm{cal} \cdot \mathrm{gm}^{-1}$ as $h_{M}$.

In the first place, we consider the effects of the water vapour at the lowest layer $\left(Z_{1+(1 / 2)}\right.$ level). Fig. 7 shows by contour lines the growth rate as a function of the relative humidity at the $Z_{1+(1 / 2)-}$

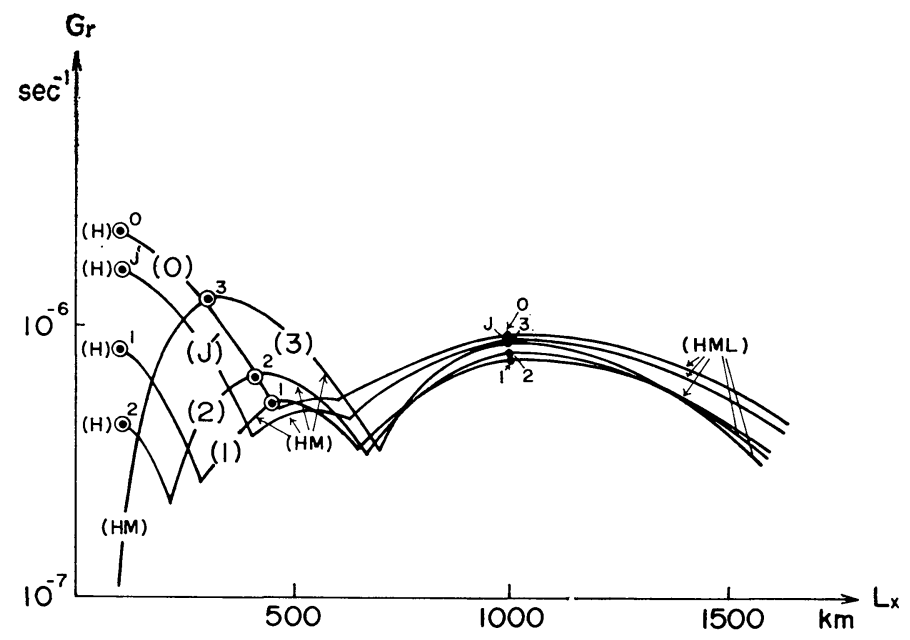

Fig. 6b Same as Fig. 3b except for case (SH-n). 


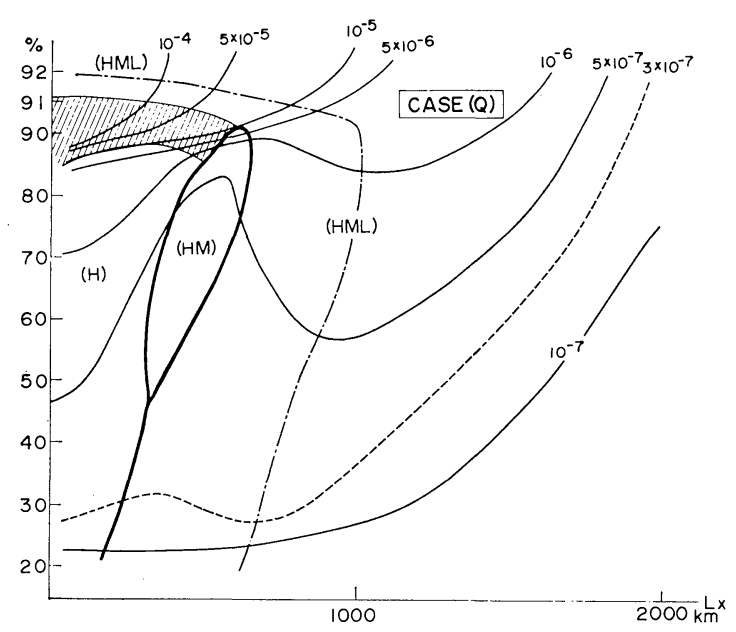

Fig. 7 Growth rate of unstable waves for case (Q). Ordinate is relative humidity at the $Z_{1+(1 / 2)}$ level and abscissa wavelength. Shaded area shows transient zone.

level and wavelength. The relative humidity at the $Z_{3}$-level is fixed to $50 \%$. The figure clearly indicates that the growth rate increases as the lowest layer becomes humid. Especially the increasing of the growth rate of mode $(\mathrm{H})$ for relatively short wavelength is most unstable. When the relative humidity in the lowest layer is below $70 \%$ or so, the growth rate of mode $(\mathrm{H})$ is comparable to those of mode (HML). However, when the relative humidity in the layer exceeds about $80 \%$, the mode $(\mathrm{H})$ becomes much unstable. Simultaneously, the range occupied by the mode $(\mathrm{H})$ extends to longer wavelength.

When the relative humidity in the lowest layer exceeds a certain value of around $90 \%$, stationary gravity waves of mode (HML) appear for shortest wavelength. And there appears a transient zone between the stationary gravity mode and the quasi-geostrophic mode where the two solutions exist overlapping each other.

In the following, we consider the effects of relative humidity at the $Z_{3}$-level. Fig. $8 \mathrm{a}$ and $8 \mathrm{~b}$ show the distribution of growth rates as a function of relative humidities at the $Z_{1+(1 / 2)}$ level and the $Z_{3}$-level for mode $(\mathrm{H})$ and mode (HML) respectively. The former is the growth rate at the wavelength of $100 \mathrm{~km}$ and the later is that of the most preferred scale of the mode. Fig. 8a indicates that the growth rate of mode $(\mathrm{H})$ rapidly increases as the both relative humidities at the $Z_{1+(1 / 2)^{-}}$and the $Z_{3}$-level become large. Especially, for the case of deep and very humid layer where relative humidities at both levels exceed $85 \%$,

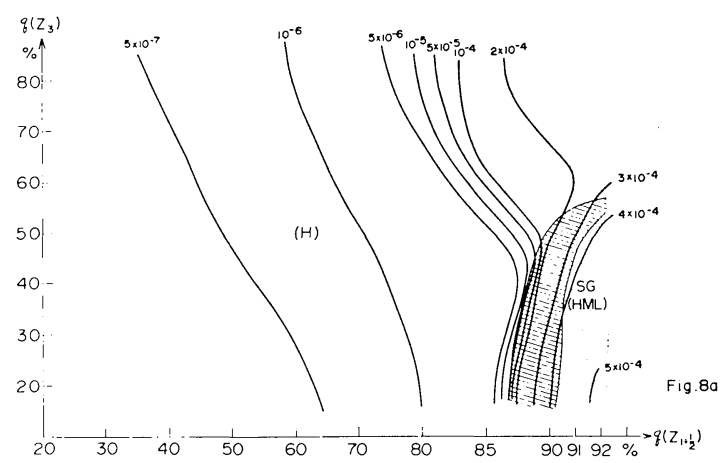

Fig. 8a Growth rates for mode $(\mathrm{H})$ at $L_{x}=100 \mathrm{~km}$ as function of relative humidity at the $Z_{3^{-}}$ level (ordinate) and at the $Z_{1+(1 / 2)}$-level (abscissa). Shaded area is transient zone and dotted area is the range of stationary gravity waves of mode (HML).

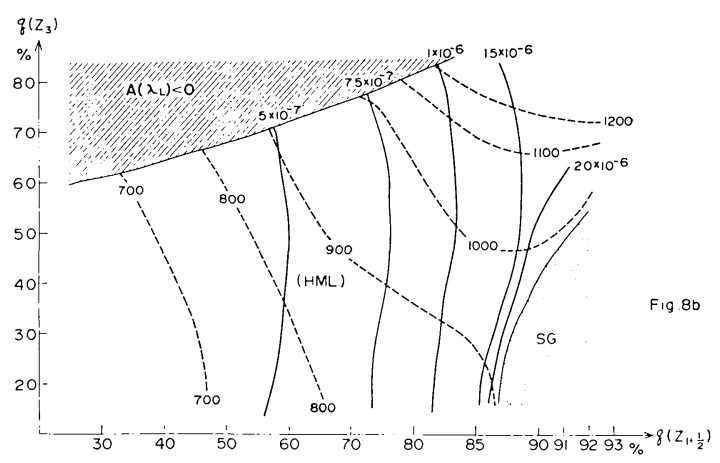

Fig. 8b Same as Fig. 8a except for mode (HML) at the most preferred scale which are indicated by dashed lines.

mode $(\mathrm{H})$ becomes very unstable. When the lowest layer is very humid but very shallow, the stationary gravity waves of mode (HML) appears.

Fig. $8 b$ indicates that the growth rates of mode (HML) are not so much affected by the relative humidity at the $Z_{3}$-level but they mainly depend on that in the lowest layer. However, the most preferred scale becomes larger as the moist layer becomes deeper. The dotted area in Fig. 8 shows the range of stationary gravity waves whose preferred scale is at the shortest wavelength.

\subsection{The effects of the cloud base condition}

As mentioned in the previous section, the time change of $h_{M}$ is not taken into consideration in the present model. So we investigate the effects of $h_{M}$ by adopting several values which satisfy Eq. (3.11) as $h_{M}$. The values of $h_{M}$ used in the following calculation and the entrainment rate corresponding to each $h_{M}$ are shown in Fig. 9a 


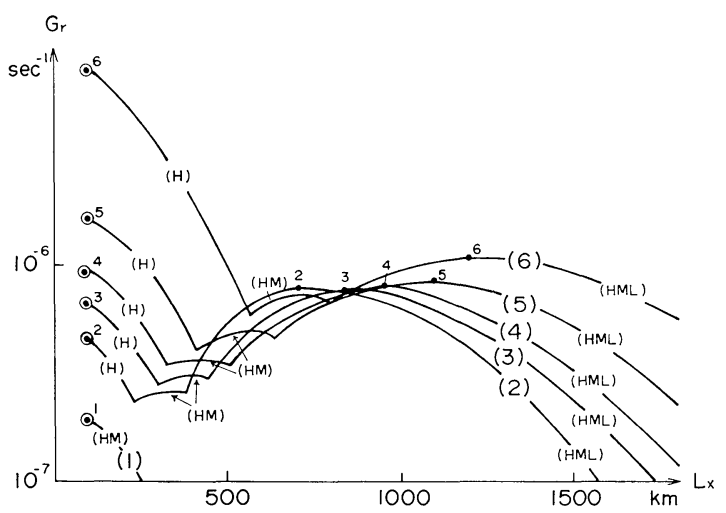

Fig. 9a Growth rates from case $\left(H_{m}-1\right)$ to case $\left(H_{m}-6\right)$.

Table 5. same as table 1 except for case $\left(H_{m}\right)$

\begin{tabular}{lrrr}
\hline CASE & $\begin{array}{c}\lambda_{H} \\
\times 10^{-5}\end{array}$ & $\begin{array}{c}\lambda_{M} \\
\times 10^{-5}\end{array}$ & $\begin{array}{c}\lambda_{L} \\
\times 10^{-5}\end{array}$ \\
\hline$H_{m}-1$ & 3.16 & 5.13 & - \\
$H_{m}-2$ & 4.80 & 7.14 & $27.70=$ case $J$ \\
$H_{m}-3$ & 5.58 & 8.10 & 29.69 \\
$H_{m}-4$ & 6.34 & 9.04 & 30.92 \\
$H_{m}-5$ & 7.77 & 10.58 & $34.61=$ case $J^{\prime}$ \\
$H_{m}-6$ & 10.19 & 13.88 & 41.45 \\
\hline
\end{tabular}

and Tabie (5) respectively. Entrainment rates of all three types increase with $h_{M}$. The stratification and the vertical distribution of water vapour are fixed as the same as those of case $(J)$.

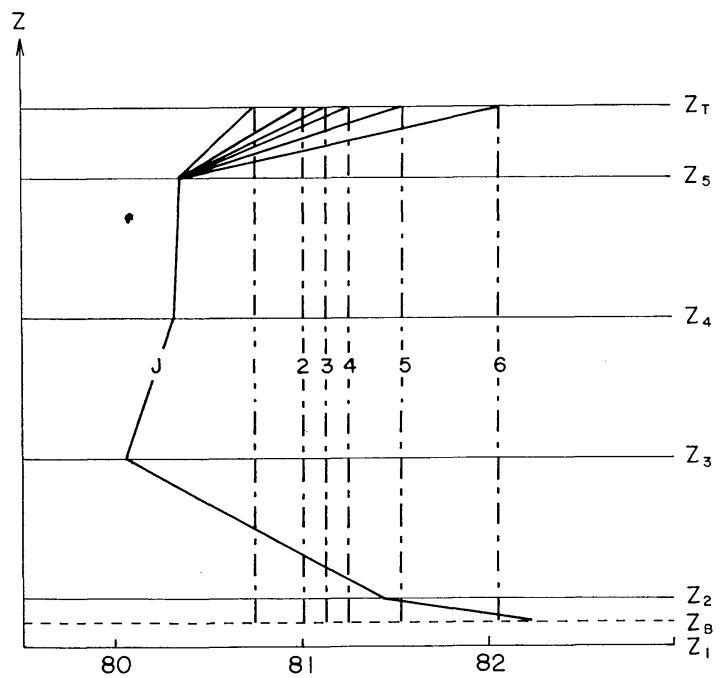

Fig. 9b The cloud base condition, $h_{M}$, from case $\left(H_{m}-1\right)$ to case $\left(H_{m}-6\right)$.

Fig. $9 \mathrm{~b}$ shows the growth rates obtained by different values of $h_{M}$. The growth rates of all modes increase with increasing $h_{M}$ as expected.
As shown in Fig. 9b, the mode $(\mathrm{H})$ is most unstable while other modes such as (HM) and (HML) have not so large growth rates. Thus we may conclude that the increasing of moist static energy of the air in the mixed layer bring the most significant effect on the mode $(\mathrm{H})$.

\section{Structures of unstable waves}

\subsection{The structure of mode $(H)$}

The structure of mode $(\mathrm{H})$ obtained for case (SLB-2) is illustrated in Fig. 10. The left part of the figure shows the vertical cross section over a half wavelength centered at minimum pressure (L) at the lowest layer, and the right part of it shows the vertical profiles of amplitudes of $M_{c}$ and $\overline{\bar{\rho}} \bar{w}$ nomalized by $\overline{\bar{\rho}}\left(Z_{1+1 / 2}\right) \bar{w}\left(Z_{1+(1 / 2)}\right)$. The phase of the maximum values of $M_{c}$ and $\overline{\bar{\rho}} \bar{w}$ are shown by arrows in the left part of figure.

We find in the figure that a marked warm and moist core exists in the upper layer. As shown in the right part of the figure, the warm core is in the layer of maximum value of $M_{c}(Z)$ which also coincides with the level of maximum large scale upward motion. Corresponding to the thermal situation, the low pressure area extending from the surface to the $Z_{4}$-level is covered by the high pressure area at the $Z_{5}$-level. Weak cold core appears in the middle layer, where large scale upward motion slightly exceeds $M_{c}$. Thus the cold area results from the excess cooling by $\overline{\bar{\rho}} \bar{w}$. In the lowest layer, there appears a very dry core, which is resulted from stronger $M_{B}$ than $\overline{\bar{\rho}}\left(Z_{1+(1 / 2)}\right) \cdot \bar{w}\left(Z_{1+(1 / 2)}\right) M_{B}$ is always larger than $\overline{\bar{\rho}}\left(Z_{1+(1 / 2)}\right) \bar{w}\left(Z_{1+(1 / 2)}\right)$ for all modes with positive growth rates. Thus the dry core in the lowest layer is a common feature. Inflow is notable in the lowest layer. This situation is consistent with the fact that the surface friction is essential for the quasi-geostrophic modes to develop.

The heat and moisture budget estimated for mode $(\mathrm{H})$ are shown in Fig. $11 \mathrm{a}$ and $11 \mathrm{~b}$ respectively. With regard to the heat budget, warming by cumulus-induced subsidence is approximately balanced by cooling caused by large scale upward motion. Cooling by evaporation of detraining liquid water from cloud is almost negligible. However, the moistening by the evaporation of detraining liquid water and detrainment of water vapour is not so small in the moisture budget. The moistening by the detrainment is about half of that by large scale upward motion in the upper layer. Then, the moist core in the upper layer shown in Fig. 10 is maintained by 

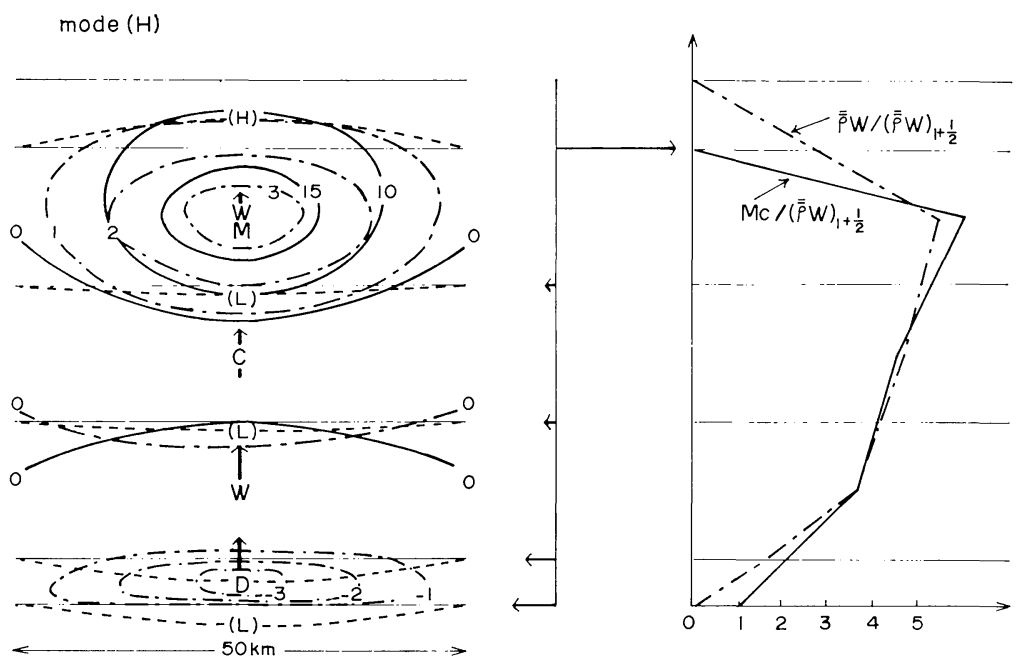

Fig. 10 The structure of mode $(H)$ which is obtained for case (SLB-2) and $L_{x}=100 \mathrm{~km}$. Solid lines, chain lines and dashed lines in the left part of the figure show $S \times 10^{3} \mathrm{cal} / \mathrm{gm} q \times 10^{3} \mathrm{~g} / \mathrm{kg}$ and $p$ respectively. Solid lines and chain lines in the right part show $M_{c} / \overline{\bar{\rho}}\left(Z_{1+(1 / 2)}\right) \bar{w}\left(Z_{1+(1 / 2)}\right)$ and $\overline{\bar{\rho}}(Z) \bar{w}(Z) / \overline{\bar{\rho}}\left(Z_{1+(1 / 2)}\right) \cdot \bar{w}\left(Z_{1+(1 / 2)}\right)$ respectivery.
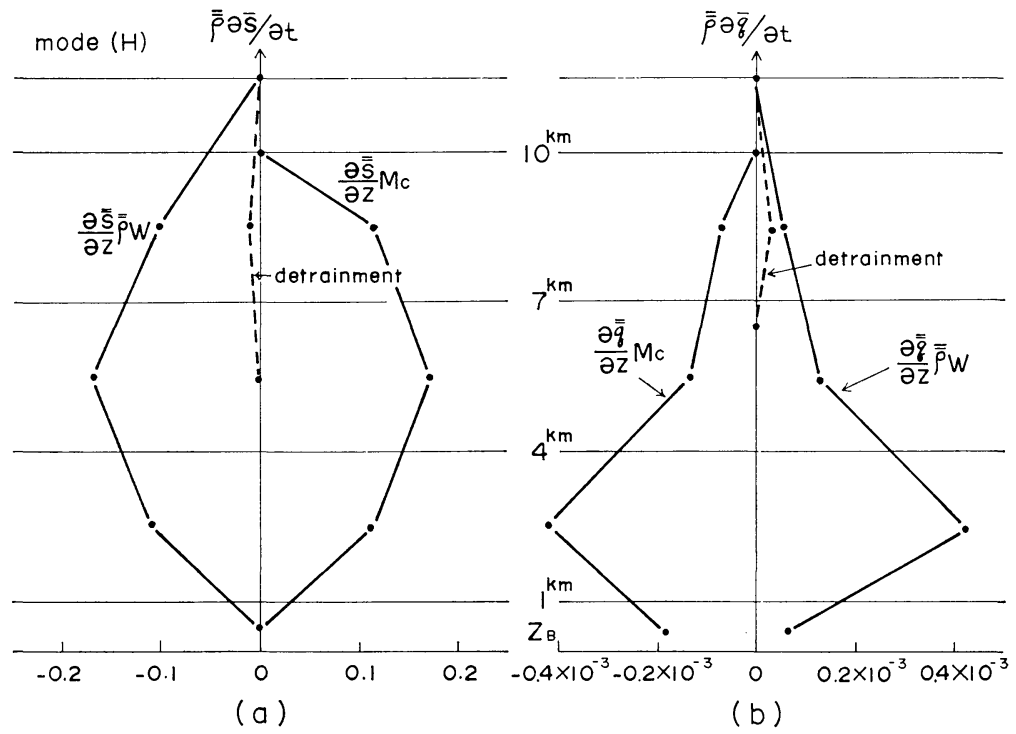

Fig. 11a The vertical profile of each term which is related to the heat budget for mode $(H)$ whose structure is shown in Fig. 10.

$11 \mathrm{~b}$ Same as Fig. 11a except to the moisture budget.

both of the detrainment and the large scale upward motion, while that in the middle layer, where there is no detrainment from clouds, is due to the excess large scale upward motion.

\subsection{The structure of mode (HM)}

The structure of mode (HM) depends on wavelength. As an example, the structure of the wave of the most preferred scale $(300 \mathrm{~km})$ for case (SLA-2) is illustrated in Fig. 12. Comparing the structure of mode (HM) with that of mode $(\mathrm{H})$, it is notable that both of the upper and the middle layers are covered by warm area. The warming in the upper layer becomes more notable than that in the middle layer, as wavelength decreases. For mode (HM), cumulus-induced subsidence is 

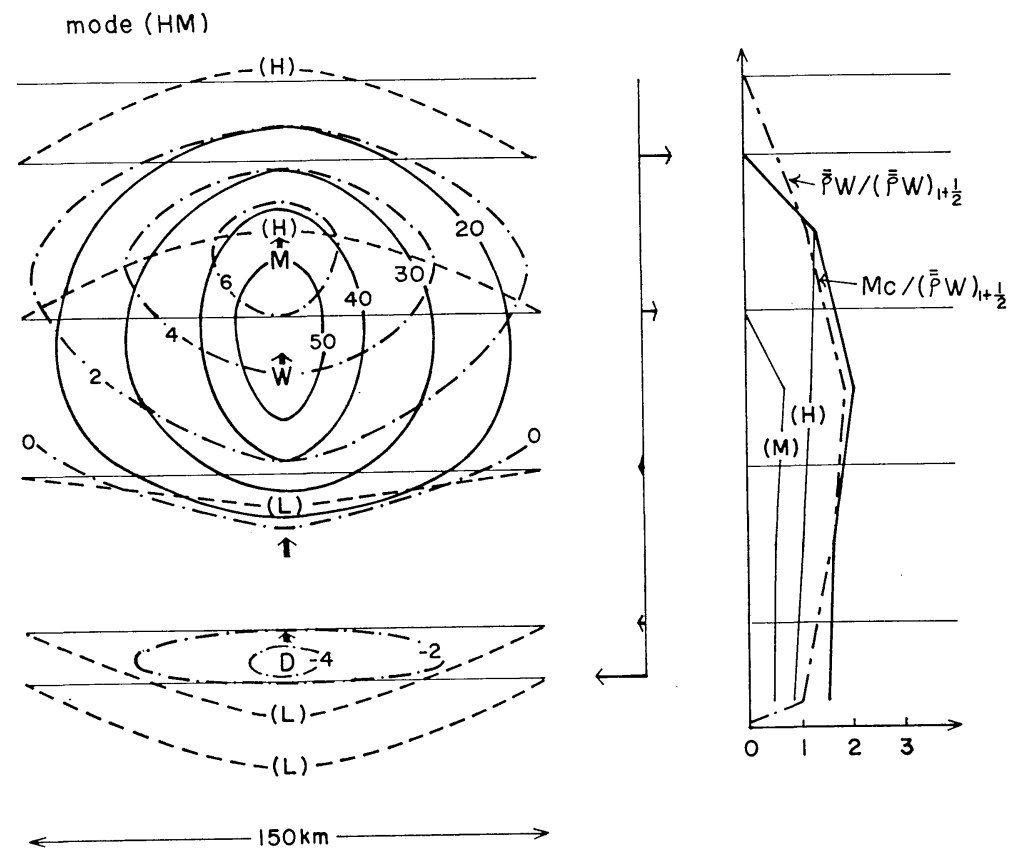

Fig. 12 Same as Fig. 10 except of mode (HM) for case (SLA-2) and $L_{x}=300 \mathrm{~km}$. Light solid lines in the right part of the figure show component of $M_{c}\left(Z \lambda_{M}\right) / \overline{\bar{\rho}}\left(Z_{1+(1 / 2)}\right) \cdot \bar{w}\left(Z_{1+(1 / 2)}\right)$ and $M_{c}\left(Z_{1} \lambda_{M}\right)$ $/ \overline{\bar{\rho}}\left(Z_{1+(1 / 2)}\right) \bar{w}\left(Z_{1+(1 / 2)}\right)$

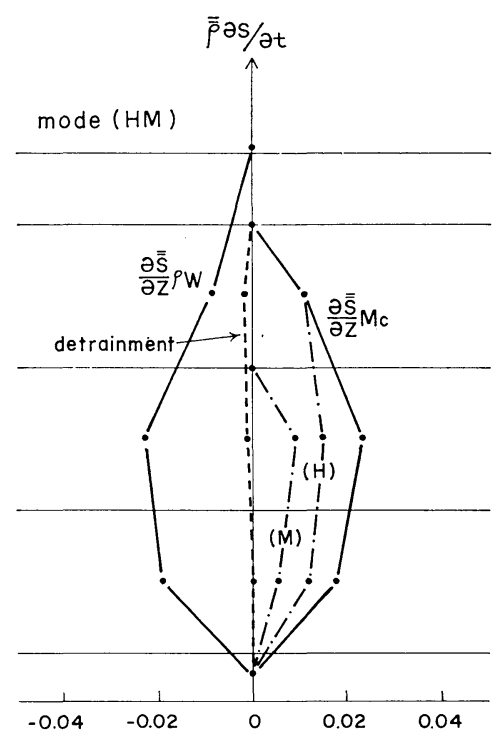

(a)

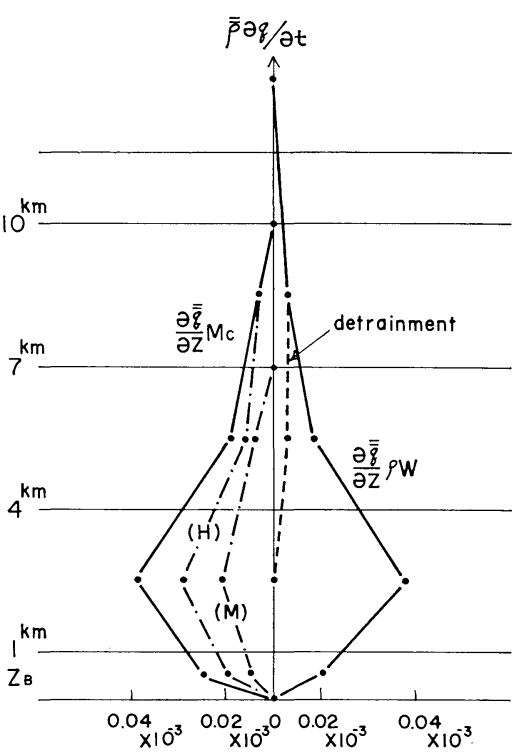

(b)

Fig. 13a Same as Fig. 11a except for wave whose structure is shown in Fig. 12.

Fig. 13b Same as Fig. 13a except to the moisture budget. 
larger than the large scale upward motion for all layers. Thus, the warming is maintained by excess cumulus-induced subsidence in all layers. As described in the later section the convective heating in the lower and the middle layers is essential for relatively small scale disturbances such as $100 \mathrm{~km}$ $500 \mathrm{~km}$, and the ratio of $m_{B}\left(\lambda_{M}\right)$ to $m_{B}\left(\lambda_{H}\right)$ increases with the increase of growth rates. Thus the warming in the middle layer tends to be intensified with increasing of growth rate. For mode (HM), the maximum of large scale upward motion is situated in the middle layer where $M_{c}$ has also a maximum value. The large scale upward motion in the upper layer is fairly weakened. This situation is due to the strong static stability in that layer. Corresponding to the warm core in the middle layer, low pressure area is confined to the relatively lower part of atmosphere.

Fig. $13 \mathrm{a}$ and $13 \mathrm{~b}$ are the heat and the moisture budgets of wave disturbance of mode (HM). The effect of the detrainment in the heat budget is quite small for mode (HM) too. The cooling by large scale upward motion is approximately balanced by the warming due to cumulus-induced subsidence by the $\mathrm{H}$ - and the M-type clouds. The effect of the detrainment in the moisture budget is fairly important. In general, the moistening by the large scale upward motion is approximately cancelled by the drying due to cumulus-induced subsidence. Thus the moistening by the detrainment is significant for the maintenance of the moist core. The moist core in the upper and the middle layer as shown in Fig. 12 is maintained by the detrainment effect of both the $\mathrm{H}$ - and the M-type clouds.

Similarly to the mode $(\mathrm{H})$, a dry core appears in the lowest layer for mode (HM) because of the excess cumulus-induced subsidence over large scale upward motion at the $Z_{B}$-level.

\subsection{The structure of mode (HML)}

In general, growth rates of mode (HML) are much smaller as compared with those of mode (H) and mode (HM). The mode (HML) becomes most unstable when the stratification and moisture field are close to the mean tropical atmosphere for hurricane season.

Fig. 14 shows the structure of mode (HML) obtained for case $(\mathbf{J})$. As shown in the figure, two maxima of static energy appear. For the case (J) the M-type cloud is weakest among three. Thus the upper one is supposed to be maintained by the $\mathrm{H}$-type, while the lower one by the L-type clouds.

For mode (HML), cumulus-induced subsidence is slightly larger than the large scale upward motion, for all layers. Thus we may consider that the warm area in all layers results from the excess cumulus-induced subsidence. Corresponding to the thermal structure, the low pressure area and hence the inflow layer are limited to a very shallow layer of the atmosphere.
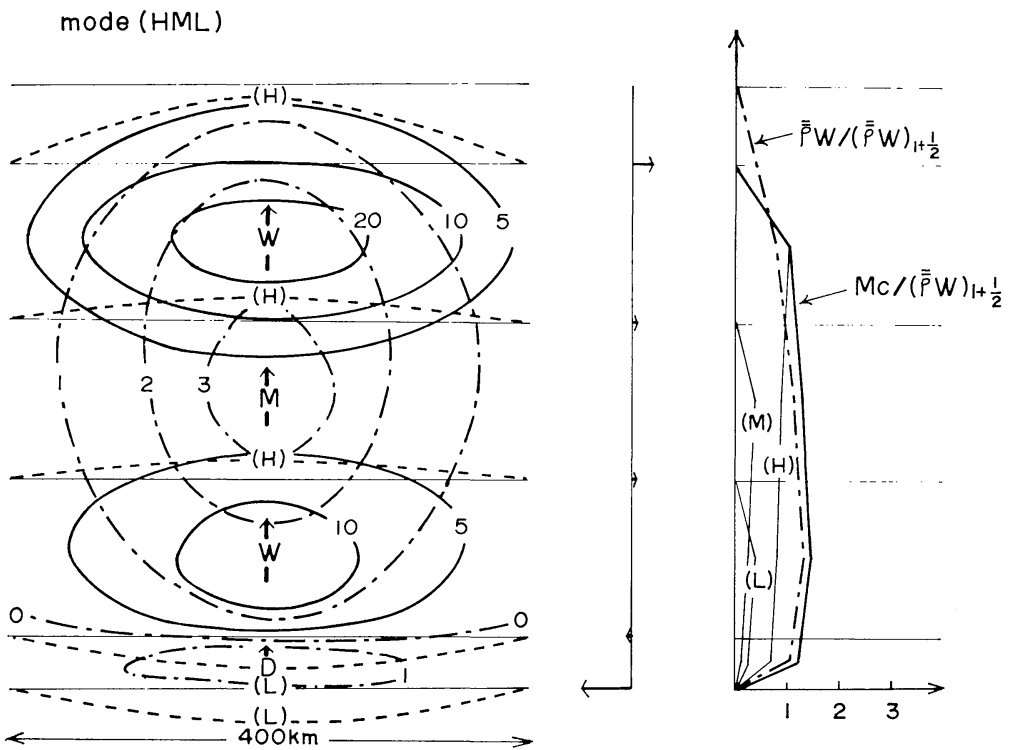

Fig. 14 Same as Fig. 10 except of mode (HML) for case (J) and $L_{x}=$ $800 \mathrm{~km}$. 


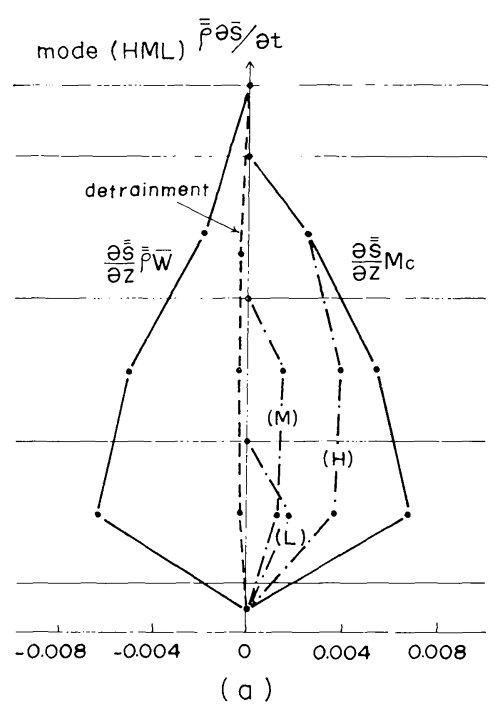

Fig. 15a Same as Fig. 11a except of wave whose structure is shown in Fig. 14.

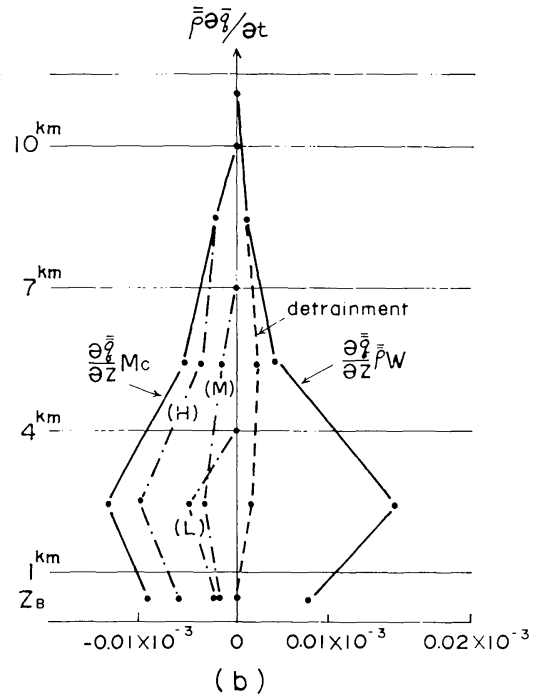

Fig. 15b Same as Fig. 15a except to the moisture budget.
Fig. $15 \mathrm{a}$ and $15 \mathrm{~b}$ show the heat and the moisture budget of mode (HML). The figure indicates that the cooling by the large scale upward motion is approximately equal to the warming by cumulus-induced subsidence and the detrainment effect is almost negligible also for mode (HML). We find in the figure that the fraction of heating by the $\mathrm{H}$-type clouds is considerably larger compared with others for all layers. This tendency becomes more notable with the increase of growth rates. As for the moisture budget, it is indicated that the detrainment effect is fairly significant for all layers except the lowest layer where no detrainment occur. These situation coincide with those of mode $(\mathrm{H})$ and mode $(\mathrm{HM})$.

\section{Discussion}

7.1 A comparison between typhoon-scale disturbance and Ooyama's typhoon mode

In the present sub-section, we shall compare the typhoon-scale disturbance described in sections 5 and 6 with those obtained by the parameterization scheme of Ooyama (1964), which may be written as follows

$$
Q_{c}(Z)=\Theta(Z) \frac{\partial \overline{\bar{S}}}{\partial Z} \rho w^{*}
$$

where $w^{*}$ is large scale upward motion at the top of the mixed layer (friction layer), and $\Theta(Z)$ is heating parameter. So far, some investigations have been made on the determination of $\Theta(Z)$ by Ooyama (1964, 1971), Yamasaki (1968) and others.

As mentioned in the previous section, the most important effect of clouds is warming by cumulusinduced subsidence. If only the warming is taken into consideration as cloud effect, the convective heating is approximately expressed by

$$
Q_{c}(Z)=\frac{\partial \overline{\bar{S}}}{\partial Z} M_{c}(Z)
$$

Comparing Eq. (7.1) with (7.2), heating parameter in Eq. (7.1) corresponds to the following quantity in this study.

$$
\Theta(Z)=M_{c}(Z) /(\overline{\bar{\rho}} \bar{w})_{1+(1 / 2)}
$$

As described in section $3, M_{c}(Z)$ is determined as an eigen solution. The ratios $M_{c}(Z) /(\overline{\bar{\rho}} \bar{w})_{1+(1 / 2)}$ are shown in Fig. 16a, 17 and 18a as functions of the static stability in the lower layer for case (SLA) and (SLB) and that in the middle layer for case (SM). The values of the ratio shown in these figures are estimated for the most preferred scale shown by $\bigcirc$ in Fig. 3b, 4b and $5 b$.

Ooyama (1964) assumed following quantities as $\Theta(Z)$

$$
\Theta(Z)=1+\frac{\theta_{e 0} *-\theta_{e 2} *}{\theta_{e 2} *-\theta_{e 1}}
$$

where $\theta_{e}$ is equivalent potential temperature and $\theta_{e}{ }^{*}$ is the saturation value. The suffices 2 and 1 denote quantities in the upper and the lower layers and 0 those in clouds. Although the model is a 


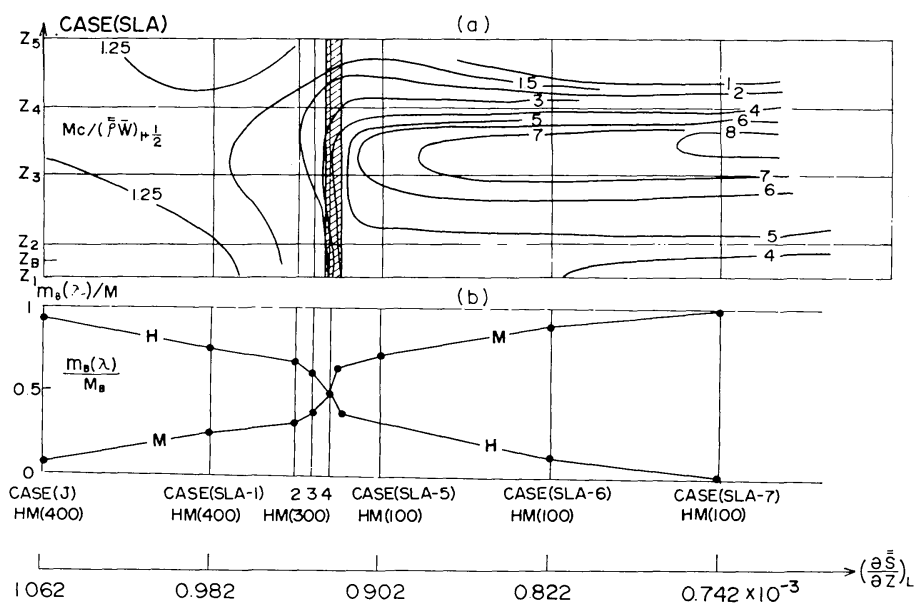

Fig. 16(a) The ratio of $M_{c}(Z) /(\overline{\bar{\rho}} w)_{1}+1 / 2$ at the most preferred scale of mode (HM) for case (SLA) as functions of the static stability in the lower layer.

Fig. 16(b) $m_{B}(\lambda) / M_{B}$ for the same case and wavelength as for Fig. (a).

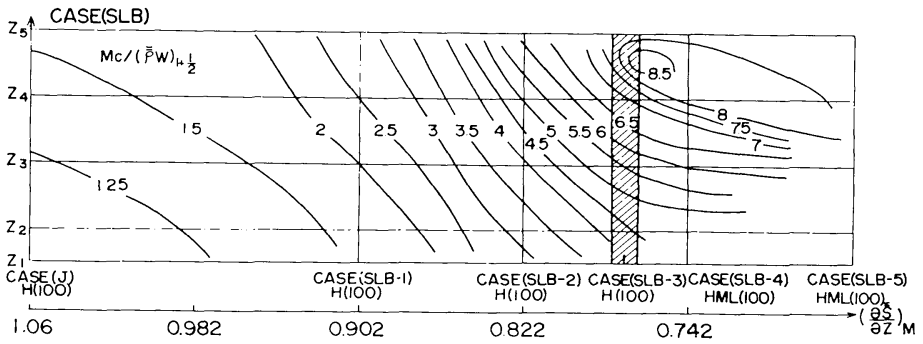

Fig. 17 Same as Fig. 16(a) except for mode (H) of case (SLB) at wavelength of $100 \mathrm{~km}$.

two-layer model including only one level as the prediction level of temperature, the assumption of Eq. (7.4) implies that the convective heating in the Ooyama's model is equivalent to the warming by cumulus-induced subsidence by clouds of a single type with an entrainment rate $\lambda^{*}$ given as

$$
\lambda^{*}=\left(\theta_{e 0}{ }^{*}-\theta_{e 2}{ }^{*}\right) /\left(\theta_{e 2}{ }^{*}-\theta_{e 1}\right) \Delta Z
$$

Ooyama's typhoon mode can be identified as mode $(\mathrm{H})$ if $\alpha$ is equal to 1

$$
\alpha=M_{B} /(\overline{\bar{\rho}} \bar{m})_{1+(1 / 2)}
$$

Fig. 17 shows that the ratio $\alpha$ of mode $(\mathrm{H})$ is slightly larger than 1 for realistic cases like case (SLB-1). The ratio $\alpha$ becomes larger as static stability in the lower layer is reduced. However, such large $\alpha$ causes strong drying in the lowest layer, which may efficiently suppress the development of a disturbance as shown in Fig. 7. Thus, mode $(\mathrm{H})$ with large $\alpha$ can not be realized in a numerical experiment where the explicit prediction of water vapour is included (cf. Rosenthal (1973)).
Growth rate of Ooyama's typhoon mode without internal friction increases with the increase of wave number although it is nearly constant for wavelength shorter than a few hundred kilometers. The characteristic features of growth rate curve and those of structure of mode $(\mathrm{H})$ in this study described in the previous section are very similar to those of Ooyama's typhoon mode.

The heating parameter $\Theta(Z)$ in Eq. (7.4) becomes larger with decreasing $\theta_{e 2} *$ under fixed values of $\theta_{e 1}$ and $\theta_{e 0^{*}}$, while the parameter becomes smaller with the decrease of $\theta_{e 1}$ under fixed values of $\theta_{e 2}{ }^{*}$ and $\theta_{e 0}{ }^{*}$ The mode $(\mathrm{H})$ tends to become dominant when the static stability in the upper layer becomes small as indicated in the cases (SH) and (SLB), while the mode tends to disappear when the lower layer becomes untable under the increased static stability in the upper layer, as shown in the cases (SLA) and (SM). Further, mode $(\mathrm{H})$ becomes unstable as the lower layer becomes humid as in the case (Q). This corresponds to increasing of $\theta_{e 1}$ under fixed values 


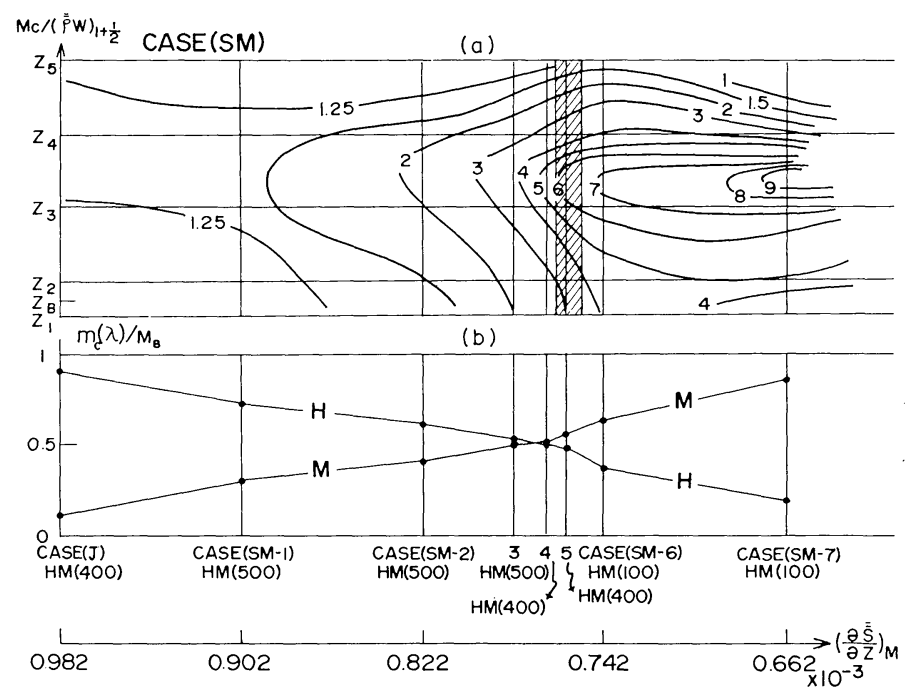

Fig. 18(a) The ratio of $M_{c}(Z) /(\overline{\bar{\rho}} \bar{w})_{1}+1 / 2$ at the most preferred scale of mode (HM) for case (SM) as functions of the static stability in the middle layer. (b) $m_{B}(\lambda) / M_{B}$ for the same case and wavelength as Fig. (a).

of $\theta_{e 2}{ }^{*}$ and $\theta_{e 0} *$. Thus we may conclude that an equivalent of the Ooyama's typhoon mode is included in our solutions that satisfy the quasiequilibrium assumption.

Syono and Yamasaki (1966) and Yamasaki $(1968,1969,1971)$ discussed the effects of vertical profile of heating parameter on properties of unstable waves in a multiple layer model by specifying functional form of $\Theta(Z)$ and it was revealed that convective heating in the lower layer is essential to the instability of typhoon-scale disturbances. According to the linear analysis of Syono and Yamasaki (1966), $\Theta\left(Z_{L}\right)$ must exceed 1 in the lower layer for a typhoon to develop. However, stationary gravity waves appear when $\Theta\left(Z_{L}\right)$ is too large to exceed a critical value. From a simplified analysis of the mechanism of slowly developing disturbances, Yamasaki (1970) found that the critical value is about $Z_{L} / Z^{*}$, where $Z_{L}$ and $Z^{*}$ are heights of the lower layer and the top of the mixed layer. Further in order to obtain unstable waves which have a typhoonlike structure, $\Theta\left(Z_{H}\right)$ in the upper part of the troposphere must be larger than $\Theta\left(Z_{L}\right)$. Thus the following criterion must be satisfied for typhoon like disturbance to develop.

$$
\begin{aligned}
& 1<\Theta\left(Z_{L}\right)<Z_{L} / Z^{*} \\
& \Theta\left(Z_{I I}\right) \geq \Theta\left(Z_{L}\right)
\end{aligned}
$$

The corresponding values of $Z_{L}$ and $Z^{*}$ in the present model are $2500 \mathrm{~m}$ and $500 \mathrm{~m}$ respectively. Thus the critical value is five or so.
In the present study it is clearly indicated in Fig. 16a and 17 that the ratio $M /(\overline{\bar{\rho}} \bar{w})_{1+(1 / 2)}$ in the lower and the middle layers is larger than unity and increases in both of the cases (SLA) and (SLB) as the static stability in the lower layer decreases and consequently the growth rate increases. This is a common feature of all developing waves described in section 5. However, the ratio in the upper layer does not increase in case (SLA). The same tendency may be recognized in case (SM) shown in Fig. 18a. Thus we may conclude that the convective heating in the lower and the middle layers play an essential role for typhoon-scale disturbance less than a few hundred kilometers in the present study, too. The shaded area in Fig. 16a, 17 and 18a show the transient zone described in section 5, and stationary gravity waves are situated at the right part to the transient zone. Fig. 16a, 17 and 18a show that the ratio of $M_{c} /(\bar{\rho} \bar{w})_{1+(1 / 2)}$ in the lower layer exceeds about 5 in the range of stationary gravity waves. These situations coincide with Yamasaki's criterion of Eq. (7.7). The ratio $M_{c} /(\overline{\bar{\rho}} \bar{w})_{1+(1 / 2)}$ in the upper layer is much larger than those in the lower layer for mode (H) in case (SLB), while the former is nearly equal to the later for mode (HM) in the case (SLA) and (SM). Thus Yamasaki's criterion of Eq. (7.8) is not so clearly realized for mode $(H M)$ as for mode $(H)$. For mode (HM) the ratio in the middle layer is very large.

Fig. $16 \mathrm{~b}$ and $18 \mathrm{~b}$ indicate the cloud mass flux 
of each type at the cloud base level normalized by total value of all types, namely $m_{B}\left(\lambda_{H}\right) / M_{B}$, $m_{B}\left(\lambda_{M}\right) / M_{B}, m_{M}\left(\lambda_{L}\right) / M_{B}$. Fig. $16 \mathrm{~b}$ shows that the fraction of M-type cloud mass flux increases with decreasing of the static stability in the lower layer. The same situation is recognized for case $(\mathrm{SM})$ in Fig. 18b. A comparison of Fig. 16a and $18 \mathrm{a}$ with $16 \mathrm{~b}$ and $18 \mathrm{~b}$ indicates that the increasing of convective heating in the lower and the middle layers results from the increase in the M-type cloud mass flux of mode (HM), in case (SLA) and (SM), while it is due to the increase in the $\mathrm{H}$-type of mode $(\mathrm{H})$ in case (SLB).

So far, Ekman pumping, and hence the surface friction has been considered as an essential factor for the development of typhoon-scale disturbance. This is quite true in the present study. If the surface friction is not taken into consideration, no solution that satisfy quasi-equilibrium assumption exist for any of seven combinations. This circumstance is consistent with the result that for quasi-geostrophic mode inflow is concentrated near the surface.

An important difference between the parameterization scheme used in this study and that of Eq. (7.1) is the fact that the convective heating in the present scheme is related to large scale upward motion not only at the top of the mixed layer but also those at all levels, viz.,

$$
\left(\begin{array}{l}
\theta_{c}\left(Z_{1+(1 / 2)}\right) \\
\theta_{c}\left(Z_{2+(1 / 2)}\right) \\
\theta_{c}\left(Z_{3+(1 / 2)}\right) \\
\theta_{c}\left(Z_{4+(1 / 2)}\right)
\end{array}\right)=\left\{Q_{c}\right\}\left(\begin{array}{l}
(\rho w)_{1+(1 / 2)} \\
(\rho w)_{2+(1 / 2)} \\
(\rho w)_{3+(1 / 2)} \\
(\rho w)_{4+(1 / 2)}
\end{array}\right)
$$

As mentioned before, matrix $\left\{Q_{c}\right\}$ is determined by a given basic field and a given cloud base condition for each mode. The matrices whose elements are normalized rowwise by the static stability of corresponding layer are shown for mode (H) and (HM) in table 6 and 7 respectively, in which typical values are adopted as the basic field parameters. In general, each element does not exceed 1 for both modes as shown in these tables. For mode (HM), large scale upward motion in the upper layer tends to suppress the convective heating in the lower and the middle layers. Cooling would be caused at any level by excess adiabatic effect due to the large scale upward motion at the level, if there were no contribution of $\overline{\bar{\rho}} \bar{w}$ at other levels, especially those at the lower levels, and a large scale disturbance can not develop. Because of contribution of $\overline{\bar{\rho}} \bar{w}$ at whole levels, convective heating at any level can becomes slightly larger than adiabatic cooling for geostrophic modes as shown in Fig. 11, 13 and 15. Note that the first column of the matrix is smaller than those of other columns by one order of magnitude in spite of the fact that Ekman suction is an essential factor for the instability. This probably implies that the surface friction is indespensable to maintain the vertical circulation in the free atmosphere which produces the convective heating being slightly larger than adiabatic cooling by $\overline{\bar{\rho}} \bar{w}$ at the level, although the direct contribution by $(\overline{\bar{\rho}} \bar{w})_{1+(1,2)}$ to the warming is much smaller quantitatively than those by $(\overline{\bar{o}} \bar{w})$ in the free atmosphere. Perhaps, it may be that the CISK mechanism included in the present parameterization is same as that in Eq. (7.1).

The ratios $\overline{\bar{\rho}} \bar{w}(Z) /(\overline{\bar{\rho}} w)_{1+(1 / 2)}, \quad m_{B}(\lambda) / M_{B}$ and $M_{B}(Z) /(\rho w)_{1+(1 / 2)}$ are shown in Fig. 19 as functions of wavelength for case (SM-4). As shown in Fig. 5b mode (HM) is most unstable with the most preferred scale at about $400 \mathrm{~km}$ for the case. Fig. 19c shows that the convective heating is much variable depending on wavelength. Be-

Table 6. matrix $\left\{Q_{c}\right\}$ for mode $(\mathrm{H})$ in case (SLB-1). $\overline{\bar{S}}_{z i+(1 / 2)}$ denotes the static stability at $Z_{i+(1 / 2)}$-level.

\begin{tabular}{l|llll:l}
\hline & $Z_{1+(1 / 2)}$ & $Z_{2+(1 / 2)}$ & $Z_{3+(1 / 2)}$ & $Z_{4+(1 / 2)}$ & \\
\hline$Q c\left(Z_{1+(1 / 2)}\right) / S Z_{1+(1 / 2)}$ & 0 & 0 & 0 & 0 & $(\rho w)_{1+(1 / 2)}$ \\
$Q c\left(Z_{2+(1 / 2)}\right) / S Z_{2+(1 / 2)}$ & 0.021 & 0.235 & 0.279 & 0.291 & $(\rho w)_{2+(1 / 2)}$ \\
$Q c\left(Z_{3+(1 / 2)}\right) / S Z_{3+(1 / 2)}$ & 0.026 & 0.291 & 0.346 & 0.360 & $(\rho w)_{3+(1 / 2)}$ \\
$Q c\left(Z_{4+(1 / 2)}\right) / S Z_{4+(1 / 2)}$ & 0.030 & 0.330 & 0.392 & 0.409 & $(\rho w)_{4+(1 / 2)}$ \\
\hline
\end{tabular}

Table 7. same as table 6 except for mode (HM)

\begin{tabular}{l|ccccc} 
& $Z_{1+(1 / 2)}$ & $Z_{2+(1 / 2)}$ & $Z_{3+(1 / 2)}$ & $Z_{4+(1 / 2)}$ & 0 \\
\hline$Q c\left(Z_{1+(1 / 2)}\right) / S z_{1+(1 / 2)}$ & 0 & 0 & 0 & 0 & $(\rho w)_{1+(1 / 2)}$ \\
$Q c\left(Z_{2+(1 / 2)}\right) / S z_{2+(1 / 2)}$ & 0.024 & 0.344 & 0.516 & -0.003 & $(\rho w)_{2+(1 / 2)}$ \\
$Q c\left(Z_{3+(1 / 2)}\right) / S z_{3+(1 / 2)}$ & 0.029 & 0.488 & 0.665 & -0.035 & $(\rho w)_{3+(1 / 2)}$ \\
$Q c\left(Z_{4+(1 / 2)}\right) / S z_{4+(1 / 2)}$ & 0.024 & 0.133 & -0.034 & 0.936 & $(\rho w)_{4+(1 / 2)}$ \\
\hline
\end{tabular}



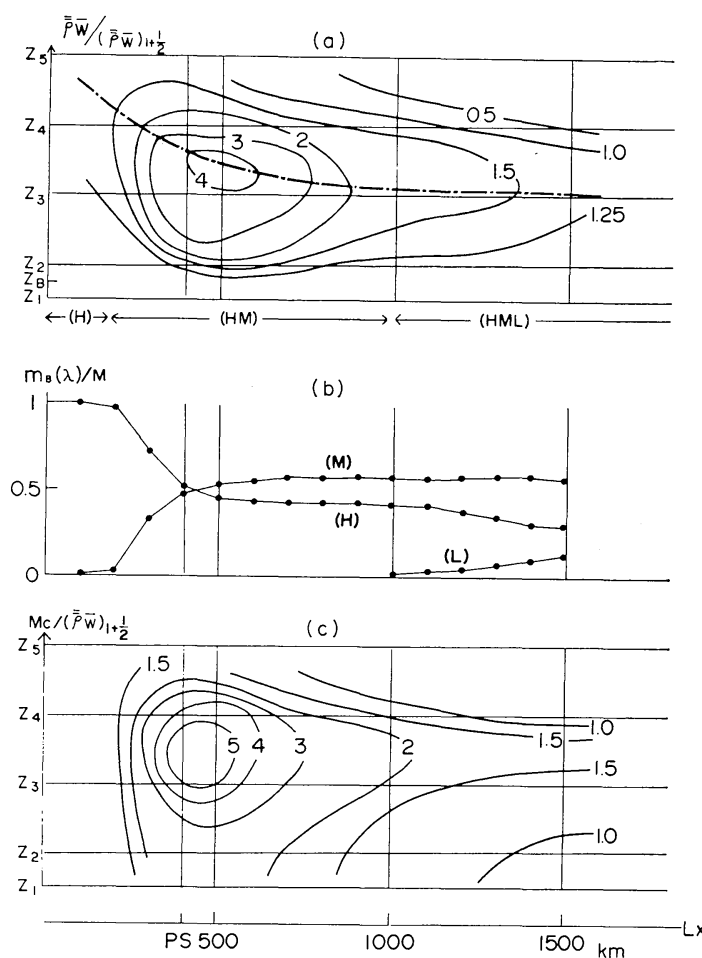

Fig. 19a Normalized large scale upward motion $\overline{\bar{\rho}}(Z) \bar{w}(Z) /(\rho w)_{1+(2 / 1)}$ as function of wavelength for case (SM-4).

Fig. 19b Normalized cloud mass flux at the $Z_{B^{-}}$ level for the three types, $m_{B}\left(\lambda_{H}\right) / M_{B}$, $m_{B}\left(\lambda_{M}\right) / M_{B}$ and $m_{B}\left(\lambda_{L}\right) / M_{B}$, as function of wavelength for the same case.

Fig. 19c The ratio of $M_{c}(Z) /(\overline{\bar{\rho}} \bar{w})_{1}+1 / 2$, as functions of wavelength for the same case.

cause matrix $\left\{Q_{c}\right\}$ does not depend on the wavelength, the scale dependency of the heating parameter $M_{c}(Z) /(\rho w)_{1+(1 / 2)}$ results from that of large scale vertical motion. In the range of mode (HM), the maximum level of large scale upward motion is lowered with wavelength and the motion in the upper layer is weakened. Corresponding to this situation the fraction of $m_{B}\left(\lambda_{H}\right)$ decreases with wavelength, while that of $m_{B}\left(\lambda_{M}\right)$ increases. This suggests that the vertical profile of $\overline{\bar{\rho}} \bar{w}$ induced in the free atmosphere is very important factor for the determination of the convective heating.

Fig. $19 \mathrm{c}$ indicates that the ratio $M_{c}(Z) /$ $(\overline{\bar{\rho}} \bar{w})_{1+1(2)}$ in the middle and the lower layer attains a maximum at the most preferred scale, while the maximum in the upper layer is not so sharply defined as in the lower layer. The maximum values of the ratio in the middle and the lower layer are located at the most preferred scale in all other cases described in section 5. For instance, in case (SLB) the maximum values are at the shortest wavelength for all layers. In the range shorter than the most preferred scale, the ratio $M_{c}(Z) /(\overline{\bar{\rho}} \bar{w})_{1+(1 / 2)}$ in the middle and the lower layer rapidly decreases with $m_{B}\left(\lambda_{M}\right)$. This is a common feature of mode (HM) with shortwave cut off.

In the parameterization scheme of Eq. (7.1), the heating parameter is prescribed and has not any scale dependency. The results shown in Fig. 19 suggest that the parameter much depends on disturbance' wavelength.

\subsection{Interpretation of mode selectivity in terms} of the quasi-equilibrium assumption

As mentioned in section 5, mode (HM) and mode $(\mathrm{H})$ become most unstable for case (SLA) and (SLB) respectively when the static stability in the lower layer is small. Fig. 20a and 20b show the ratio $\overline{\bar{\rho}} \bar{w}(Z) / \overline{\bar{\rho}} \bar{w}\left(Z_{1+(1 / 2)}\right)$. These values are estimated at the same wavelength as those in Fig. 16 and 17.

Comparing Fig. 20a with 20b, it is noted that the large scale upward motion in the lower and the middle layer becomes intense in both cases as the static stability in the lower layer decreases, while the motion in the upper layer is much suppressed in case (SLA) than in case (SLB). The suppression in the former case may be mainly due to increased static stability in the upper layer, and $\mathrm{H}$-type clouds are difficult to develop in spite of reduced static stability of the lower layer. Thus M-type clouds become necessary to cancel the effect of large scale upward motion to increase $A\left(\lambda_{M}\right)$ and $A\left(\lambda_{L}\right)$. Fig. 16b shows that the fraction of M-type rapidly increases with growth rate. However, H-type clouds are not suppressed for case (SLB), because the static stability in the upper layer is not increased. Then, H-type becomes intense as the lower layer becomes unstable. Then the increase of $A\left(\lambda_{M}\right)$ and $A\left(\lambda_{L}\right)$ by $\overline{\bar{\rho}} \bar{w}$ can be cancelled by cumulus-induced subsidence from the $\mathrm{H}$-type. Thus the mode $(\mathrm{H})$ becomes most unstable for this case.

Comparison of corresponding pairs in case (SLA) and (SLB) in which the static stability in the lower and the middle layer is same, such pair like case (SLA-5) and (SLB-1), case (SLA-6) and (SLB-2) and case (SLA-7) and (SLB-4), shows that the ratio $M_{c}(Z) /(\overline{\bar{\rho}} \bar{w})_{1+(1 / 2)}$ in the lower layer and middle layer of case (SLA) is larger than that of case (SLB) (cf. Fig. 16a ard 17). Growth rate of mode $(\mathrm{HM})$ in the former case is also larger 
(a)

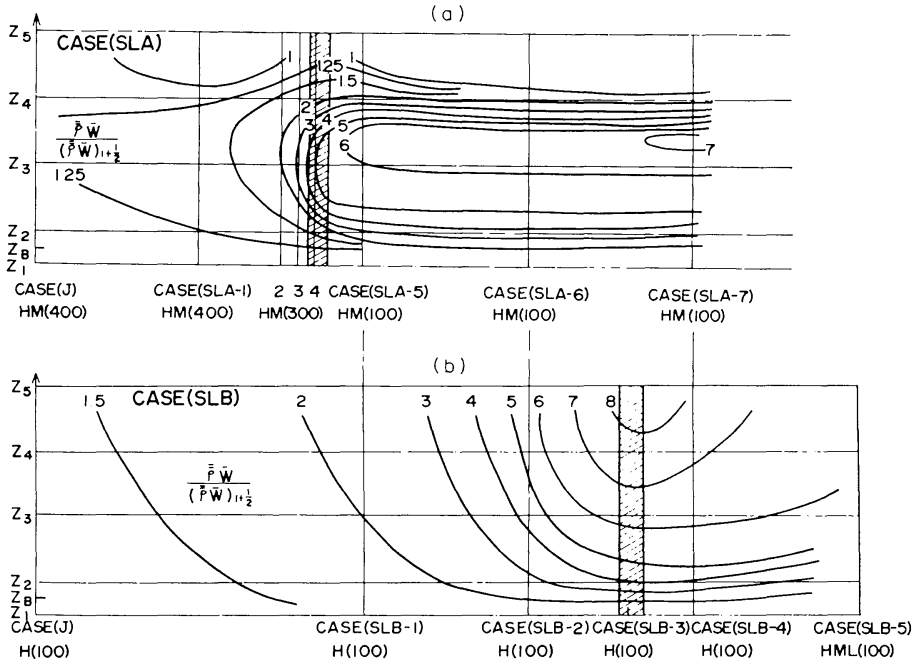

Fig. 20(a) $\overline{\bar{\rho}}(Z) \bar{w}(Z) /(\overline{\bar{\rho}} \cdot \bar{w})_{1+(1 / 2)}$ for case (SLA) at the most preferred scale of mode (HM) as functions of the static stability in the lower layer. (b) Same as Fig. (a) except of mode $(\mathrm{H})$ for case (SLB).

than that of mode $(\mathrm{H})$ in the later case. This suggests that $\mathrm{M}$-type clouds are more responsible for the heating in the lower and the middle layer than H-type. Thus we may suppose that the stable upper layer is rather favorable for typhoon-scale disturbance to develop, if the lower and the middle layer is unstable.

As described in sub-section (5.2), the typhoonscale disturbance becomes most unstable as relative humidity in the lowest and the lower layer is increased. The disturbance belongs to mode (H). The situation is similar to case (SLB) mentioned above. The increased relative humidity in these layers does not cause suppression of large scale upward motion in the upper layer. Hence, $\mathrm{H}$-type clouds become strong with increasing relative humidity because the increase of cloud work function by the moistening effect of large scale upward motion is intensified with increasing of $\partial \overline{\bar{q}} / \partial Z$ in the lower layer. When the lowest layer is very humid but shallow, namely $\partial \overline{\bar{q}} / \partial Z$ in the lower layer is extremely large, stationary gravity waves of mode (HML) appear. This indicates that the increase of $A\left(\lambda_{M}\right)$ and $A\left(\lambda_{L}\right)$ by the moistening effect of large scale upward motion in the lower layer can not be cancelled by drying effect by cumulus-induced subsidence from $\mathrm{H}$-type only.

The effects of the stratification and the vertical distribution of water vapour on the convective heating are discussed in the present sub-section. However the effect of increasing of entrainment rate is involved implicitly in all cases, although we can not separate the effect from the former ones.

\section{Concluding remarks}

As a theoretical basis for numerical simulation of a developing tropical cyclone, we have examined properties of unstable waves produced by the CISK mechanism using the parameterization scheme proposed by Arakawa-Schubert (1974). Characteristic features of unstable waves, as revealed by the present linear analysis, are summarized below.

(1) In general, the cloud types accompanying with a large scale disturbance is uniquely determined for a wavelength and for a given basic field including the mixed layer.

(2) Mode (H), (HM) and (HML) appear in turn with increasing wavelength, although the mode which becomes most unstable, depends on the basic field. For short wavelength only tall clouds such as H-type can exist. The overstabilization would occur if the lower clouds existed. On the other hand, for long wavelength where taller clouds are difficult to dominant, lower clouds such as M- and L-type are required to maintain quasi-equilibrium.

(3) As the lower layer becomes unstable and humid, a typhoon-scale disturbance whose preferred scale is less than a few hundred kilometers becomes most unstable. The disturbance belongs to either mode (HM) or mode $(\mathrm{H})$. 
When the static stability in the upper layer is large, mode (HM) whose preferred scale is at about $500 \mathrm{~km}$ becomes most unstable, while when the static stability in the layer is small, mode $(\mathrm{H})$ whose preferred scale is at the shortest wavelength tends to dominate.

(4) The surface friction is essential to unstable waves which satisfy 'quasi-equilibrium assumption'.

(5) The convective heating in the lower layer seems to be essential for the typhoon-scale disturbance. The heating in the layer increases with growth rate.

(6) When the convective heating in the lower layer exceeds a critical value of about five, unstable stationary gravity waves whose growth rate is larger than Coriolis parameter at the shortest wavelength, appear.

(7) Mode $(\mathrm{H})$ can be approximately identified as the typhoon mode of Ooyama (1964).

Among many simplifications used in the present study, the most controversial assumption may be that of 'unconditional heating'. There is also an unresolved question concerning propagating gravity waves. The propagating waves are outside the scope of the present linear model because of the difficulty in the methodology. However, propagating waves may become important especially when the vertical wind shear is included in the basic field. It is desired to investigate the effect of vertical shear and the interaction between stationary waves and propagating waves by a numerical experiment in near future.

Only three cloud types are considered in the present model and mode (H), (HM) and (HML) in the present results seem to be too crude for a representation of the real atmosphere in which many cloud types are possible. We will discuss more continuous transition of modes using a multiple layer model in next paper.

\section{Acknowledgments}

The author is grateful to Professor R. Yamamoto of Kyoto University for his encouragement through this work. The author wishes to express special thanks to Dr. M. Yamasaki of Meteorological Research Institute for his valuable discussions and comments. The author would like to express her sincere gratitude to Dr. K. Ooyama of National Center for Atmospheric Research for his helpful comments and suggestions for the original manuscript.

\section{Appendix A}

Cloud work function of $\lambda$-type, $A(\lambda)$, and time derivative of it are expressed as follows by using the assumption (2) and (3).

$$
\begin{aligned}
A(\lambda)=\int_{Z_{B}}^{Z_{D}(\lambda)} \eta(Z, \lambda)\left[\overline { \overline { \rho } } ( Z ) \beta ( Z ) \left\{h_{c}(Z, \lambda)\right.\right. & \\
- & \left.\left.\bar{h}^{*}(Z)\right\}+g \delta\left\{\bar{q}^{*}(Z)-\bar{q}(Z)\right\}\right] d Z \\
\frac{\partial A(\lambda)}{\partial t}= & \int_{Z_{B}}^{Z_{D}(\lambda)} \eta(Z, \lambda) \frac{\partial}{\partial t}[\overline{\bar{\rho}}(Z) \beta(Z) \\
& \times\left\{h_{c}(Z, \lambda)-\bar{h}^{*}(Z)\right\} \\
& \left.+g \delta\left\{\bar{q}^{*}(Z)-\bar{q}(Z)\right\}\right] d Z \\
& -\lambda \frac{\partial Z_{B}}{\partial t} A(\lambda)-\frac{\partial Z_{B}}{\partial t} \\
& \times\left\{\overline{\bar{\rho}}\left(Z_{B}\right) \alpha\left(Z_{B}\right)\left(S_{v c}\left(Z_{B} \lambda\right)-\bar{S}_{v}\left(Z_{B}\right)\right)\right\}
\end{aligned}
$$

where

$$
\begin{aligned}
& \delta=0.608 \\
& h=C_{p} T+g Z+L q \\
& \overline{\bar{\rho}}(Z) \alpha(Z)=g / C_{p} \overline{\bar{T}}(Z) \\
& \begin{aligned}
\overline{\bar{\rho}}(Z) \beta(Z)= & \left(g / C_{p} \overline{\bar{T}}(Z)\right)(1+\delta \gamma(Z) \varepsilon(Z)) / \\
& \quad \times(1+\gamma(Z))
\end{aligned} \\
& \begin{aligned}
\varepsilon(Z)=C_{p} \overline{\bar{T}}(Z) / L \\
\gamma(Z)=L / C_{P}\left(\partial \overline{\bar{q}}^{*}(Z) / \partial T\right)
\end{aligned}
\end{aligned}
$$

Cloud terms in Eq. (A.2) are expressed by variables of large scale disturbance and basic field under a given cloud base condition by using the assumption (1).

$$
\begin{aligned}
h_{c}(Z, \lambda)= & (1 / \eta(Z, \lambda))\left\{h_{c}\left(Z_{B}, \lambda\right)\right. \\
+ & \left.\lambda \int_{Z_{B}}^{Z} \eta\left(Z^{\prime}, \lambda\right) \overline{\bar{h}}\left(Z^{\prime}\right) d Z^{\prime}\right\} \quad \text { (A. 3) } \\
\frac{\partial h_{c}(Z, \lambda)}{\partial t}= & (1 / \eta(Z, \lambda))\left\{\lambda \int_{Z_{B}}^{Z^{\prime}} \eta(Z, \lambda) \frac{\partial \bar{h}(Z)}{\partial t} d Z\right. \\
& +\lambda \frac{\partial Z_{B}}{\partial t}\left(h_{c}\left(Z_{B} \lambda\right)-\overline{\bar{h}}\left(Z_{B}\right)\right\}
\end{aligned}
$$

$$
\begin{aligned}
& \int_{Z_{B}}^{Z_{D}(\lambda)} \eta(Z, \lambda) \frac{\partial}{\partial t}\left\{\overline{\bar{\rho}}(Z) \beta(Z) h_{c}(Z, \lambda) d Z\right. \\
= & \lambda \int_{Z_{B}}^{Z_{D}(\lambda)} \eta(Z, \lambda) \frac{\partial \bar{h}(Z)}{\partial t} \overline{\bar{\rho}}(Z) b(Z) d Z \\
& -\lambda \frac{\partial Z_{b}}{\partial t} \overline{\bar{\rho}}\left(Z_{B}\right) b\left(Z_{B}\right) \Delta h
\end{aligned}
$$

Substituting Eq. (A.3)-Eq. (A.5) into Eq. (A.2), we obtain

$$
\begin{aligned}
\frac{\partial A(\lambda)}{\partial t}= & \int_{Z_{B}}^{Z_{D}(\lambda)} \eta(Z, \lambda)\left\{-\alpha(Z) \frac{\partial S_{v}(Z)}{\partial t}\right. \\
& \left.+\lambda b(Z, \lambda) \frac{\partial \bar{h}(Z)}{\partial t}\right\} \overline{\bar{\rho}}(Z) d Z
\end{aligned}
$$




$$
\begin{aligned}
& -\overline{\bar{\rho}}\left(Z_{B}\right) \frac{\partial Z_{B}}{\partial t}\left(-\alpha\left(Z_{B}\right) \Delta S_{v}\right. \\
& \left.+\lambda b\left(Z_{B} \lambda\right) \Delta h+\dot{\lambda} \frac{A(\lambda)}{\bar{\rho}\left(Z_{B}\right)}\right)
\end{aligned}
$$

where

$$
\begin{aligned}
& \overline{\bar{\rho}}(Z) b(Z)=\int_{Z}^{Z_{D}(\lambda)} \overline{\bar{\mu}}\left(Z^{\prime}\right) \beta\left(Z^{\prime}\right) d Z^{\prime} \\
& \Delta S_{v}=\overline{\bar{S}}_{v}\left(Z_{B}\right)-S_{v M} \\
& \Delta h=\overline{\bar{h}}\left(Z_{B}\right)-h_{M}
\end{aligned}
$$

The time derivative terms in Eq. (A.4) are written as follows

$$
\begin{aligned}
\frac{\partial \bar{S}_{v}(Z)}{\partial t}= & -D(Z) L \hat{l}_{c}(\lambda)(1-\varepsilon(Z) \delta) \\
& +M_{c}(Z) \frac{\partial \overline{\bar{S}}_{v}}{\partial Z}-\overline{\bar{\rho}}(Z) \bar{w}(Z) \frac{\partial \overline{\bar{S}}_{v}}{\partial Z} \\
\frac{\partial \bar{h}(Z)}{\partial t}= & -D(Z)\left\{\left(h_{c}(\lambda)-\overline{\bar{h}}^{*}(Z)\right)+L\left(\overline{\bar{q}}^{*}(Z)\right.\right. \\
& -\overline{\bar{q}}(Z))\}+M_{c}(Z) \frac{\partial \overline{\bar{h}}}{\partial Z} \\
& -\overline{\bar{\rho}}(Z) \bar{u}(Z) \frac{\partial \overline{\bar{h}}}{\partial Z} \\
\frac{\partial Z_{B}}{\partial t}= & -M_{B}+\overline{\bar{\rho}}\left(Z_{B}\right) \bar{w}\left(Z_{B}\right)
\end{aligned}
$$

Finally, we can obtain the simplified form of $A(\lambda) / \partial t$, i.e., Eq. (2.14) by substituting Eq. (A.7)-(A.9) into Eq. (A.6). Here, $K_{D}, K_{V}, K_{\text {MIX }}$ and $F_{c}$ are expressed as follows.

$$
\begin{aligned}
K_{D}\left(\lambda, \lambda^{\prime}\right)= & \eta\left(Z_{D^{\prime}}, \lambda^{\prime}\right) \eta\left(Z_{D}, \lambda\right) \\
& \times\left[\left(1-\varepsilon\left(Z_{D}^{\prime}\right) \delta\right) \alpha\left(Z_{D}^{\prime}\right) L \hat{l}_{c}\left(\lambda^{\prime}\right)\right. \\
& +\lambda b\left(Z_{D_{1}}, \lambda\right)\left\{\left(\hat{h}_{c}\left(\lambda^{\prime}\right)-\overline{\bar{h}}^{*}\left(Z_{D}{ }^{\prime}\right)\right)\right. \\
& \left.\left.-L\left(\overline{\bar{q}}^{*}\left(Z_{D}^{\prime}\right)-\overline{\bar{q}}\left(Z_{D}\right)\right)\right\}\right] \\
K_{v}\left(\lambda, \lambda^{\prime}\right)= & \int_{Z_{B}}^{Z_{D}(\lambda)} \eta(Z, \lambda) \eta\left(Z, \lambda^{\prime}\right) \\
& \times\left\{-\alpha(Z) \frac{\partial \overline{\bar{S}_{v}}}{\partial Z}+\lambda b\left((Z, \lambda) \frac{\partial \overline{\bar{h}}}{\partial Z}\right\} d Z\right. \\
K_{\mathrm{MIX}}(\lambda)= & -\alpha\left(Z_{B}\right) \Delta S_{v}+\lambda k\left(Z_{B}, \lambda\right) \Delta h \\
& +\lambda A(\lambda) / \overline{\bar{\mu}}\left(Z_{E}\right) \\
F_{c}(Z, \lambda)= & \eta(Z, \lambda)\left(-\alpha(Z) \frac{\partial \overline{\bar{S}_{v}}}{\partial Z}+\lambda b(Z, \lambda) \frac{\partial \overline{\bar{h}}}{\partial Z}\right)
\end{aligned}
$$

where $Z_{D}^{\prime}$ is the detrainment level of $\lambda^{\prime}$-type.

\section{Appendix B}

Elements of matricies $\left\{\boldsymbol{K}_{D}\right\},\left\{\boldsymbol{K}_{V}\right\},\left\{K_{M I X}\right\},\left\{\boldsymbol{F}_{C}\right\}$ and $\left\{F_{M}\right\}$ are given as follows in the present finite difference scheme.

$$
K_{D n m}=K_{D}\left(\lambda_{n}, \lambda_{m}\right)=\eta\left(\lambda_{m}\right) X\left(Z_{I D}, \lambda_{n}\right)
$$

where $Z_{I D}$ is the detrainment level of the $\lambda_{m}$-type and $I D=5-m+1$, and

$$
\begin{aligned}
& X\left(Z_{I D}, \lambda_{n}\right)= \alpha\left(Z_{I D}\right) L \hat{l}_{c}\left(\lambda_{m}\right)\left(1-\varepsilon\left(Z_{I D}\right) \delta\right) \\
&+\lambda_{n} b\left(Z_{I D} \lambda_{n}\right)\left\{\hat{h}_{c}\left(\lambda_{m}\right)-\overline{\bar{h}}^{*}\left(Z_{I D}\right)\right. \\
&\left.\left.+L\left(\overline{\bar{q}}^{*}\left(Z_{I D}\right)-\overline{\bar{q}}\left(Z_{I D}\right)\right)\right\}\right] \\
& \times \frac{\left(\eta\left(Z_{I D+(1 / 2)}, \lambda_{n}\right)+\eta\left(Z_{I D-(1 / 2)}, \lambda_{n}\right)\right.}{2} \\
& K_{v n m}=K_{v}\left(\lambda_{n}, \lambda_{m}\right) \\
&=\sum_{i=1}^{I D-I} \eta\left(Z_{i+(1 / 2)}, \lambda_{m}\right) Y\left(Z_{i+(1 / 2)}, \lambda_{n}\right)
\end{aligned}
$$

where

$$
\begin{aligned}
& Y\left(Z_{i+(1 / 2)}, \lambda_{n}\right)=\eta\left(Z_{i+(1 / 2)}, \lambda_{n}\right) \\
& \times\left\{\left.\lambda_{n} b\left(Z_{i+(1 / 2)}, \lambda_{n}\right) \frac{\partial \overline{\bar{h}}}{\partial Z}\right|_{i+(1 / 2)}\right. \\
& \left.-\alpha\left(Z_{i+(1 / 2)}\right) \frac{\partial Z_{i+(1 / 2)}}{\partial S_{v}} \mid\right\}\left(Z_{i+1}-Z_{i}\right) \\
& Y\left(Z_{i+(1 / 2)}, \lambda_{n}\right)=0 \quad(i>5-n) \quad(i \leqq 5-n) \\
& K_{\text {M IX }}\left(\lambda_{n}\right)=\left(-\alpha\left(Z_{1+(1 / 2)}\right) \Delta S_{v}\right. \\
& +\lambda_{n} b\left(Z_{1+(1 / 2)}, \lambda_{n}\right) \Delta h \\
& \left.+\lambda_{n} A\left(\lambda_{n}\right) / \rho\left(Z_{1+(1 / 2)}\right)\right) \\
& F_{c i n}=F_{c}\left(Z_{i+(1 / 2)}, \lambda_{n}\right)=\eta\left(Z_{i+(1 / 2)}\right) \\
& \times\left\{\lambda_{n} b\left(Z_{i+(1 / 2)}, \lambda_{n}\right) \frac{\partial \overline{\bar{h}}}{\partial Z_{i+(1 / 2)}} \mid\right. \\
& \left.-\alpha\left(Z_{i+(1 / 2)}\right) \frac{\partial S_{v}}{\partial \bar{Z}_{i+(1 / 2)}}\right\}\left(Z_{i+1}-Z_{i}\right)
\end{aligned}
$$

$$
F_{m}\left(\lambda_{n}\right)=K_{\mathrm{MIX}}\left(\lambda_{n}\right)
$$

\section{References}

Arakawa, A., 1969: Parameterization of cumulus convection. Proc. WMO/IUGG Symposium on Num. Wea. Pred. In Tokyo, pp. IV. 8, 1-6. and Schubert, W. H., 1974: Interaction of cumulus cloud ensemble with the large scale environment. Part I, J. Atmos. Sci., 31, 674-701. , Katayama, A., Schubert, W. H., Tokioka, T., Schleisnger, M., Chao, M., Randall, D., and Lord, S.: The UCLA atmospheric general circulation model.

Charney, J. G., and Eliassen, A., 1964: On the growth of the hurricane depression. J. Atmos. Sci., 21, 68-75.

Jordan, C. L., 1958: Mean soundings for the West Indies area. J. Meteor., 15, 91-97.

Kuo, H. L., 1965: On the formation and intensification of tropical cyclone through latent heat by cumulus convection. J. Atmos. Sci., 22, 40-63.

Kurihara, Y., 1973: A scheme of moist convective adjustment. Mon. Wea. Rev., 101, 547-553. 1975: Budget analysis of a tropical cyclone simulated in an axisymmetric numerical model. J. Atmos. Sci., 32, 25-59.

, and Tuleya, R. E., 1974: Structure of a tropical cyclone developed in a three-dimensional 
numerical simulation model. J. Atmos. Sci., 31, 893-919. Angular momentum budget of a three-dimensional tropical cyclone model. J. Atmos. Sci., 32, 287 301.

Manabe, S., Smagorinsky, J., and Stricker, R. F., 1965: Simulated climatorogy of a general circulation model with a hydrologic cyclone. Mon. Wea. Rev., 93, 769-798.

Ooyama, K., 1964: A dynamical model for the study of tropical cyclone development. Geofisca International (Mexico), 4, 187-198.

1969: Numerical simulation of the life cycle of tropical cyclone development. J. Atmos. Sci., 26,

1971: A theory on parameterization of cumulus convection. J. Met. Soc., 49, special issue 744-756.

Rothenthal, S., 1970: A circularly symmetric primitive equation model of a tropical cyclone development containing an explicit water vapour cycle. Mon. Wea. Rev., 98, 643-663.

, 1973: Hurricane modeling experiments with a new parameterization for cumulus con- vection. NOAA Tech. Memorandum ERL WMPO-4.

Syono, S., and Yamasaki, 1966: Stability of symmetrical motion driven by latent heat released by cumulus convection under the existence of surface friction. J. Met. Soc. Japan, 44, 353-375.

Yamasaki, M., 1968a: Numerical simulation of tropical cyclone development with the use of primitive equation. J. Met. Soc. Japan, 46, 178 201.

1968b: A tropical cyclone model with parameterized vertical partion of released latent heat. J. Met. Soc. Japan, 46, 202-214.

1968c: Detailed analysis of a tropical cyclone simulated with a 13-layer model. Pap. Met. Geophys., 19, 559-585.

, 1969: Large scale disturbances in the conditionally unstable atmosphere in low latitude. Pap. Met. Geophys., 20, 289-336.

Yamasaki, M., 1970: Dynamics of typhoon development (Recent theory and numerical experiment). Tenki, 17, 207-227. (in Japanese)

, 1971: Further study of wave disturbances in the conditionally unstable model tropics. $J$. Met. Soc. Japan, 49, 391-415.

\title{
Arakawa-Schubert パラメタリゼーションにより生ずる 台風スケールの擾乱の性質
}

\author{
和田美鈴 \\ 気象研究所
}

台風の発生及び発達機構に関する理論的基礎を得るため, 線型不安定論により Arakawa-Schubert (1974) によっ て提案されたパラメタリゼーションから生ずる不安定波の性質を調べた。

用いられたモデルは自由大気層として 3 層, 混合層として 1 層を含む 4 層モデルである。したがってH型，M型， L 型の 3 つの型の積雲を定義出来る。このモデルでは, 積雲の共存に関連して次の 7 通りの組合せを考えることが出 来る; (HML), (HM), (HL), (ML), (H), (M), (L).

これら 7 通りの組合せのらち，大規模擾乱に伴い発現するものは，擾乱のスケールと擾乱をとりをく基本場の状態 を与えれば，汇とんど全ての場合について固有解として一意的に決定しらる。一般に擾乱のスケールが増すにしたが って, mode $(\mathrm{H})$, mode (HM), mode (HML) の順番で現れる。しかしこれら 3 つの mode のうち, どれが最も 卓越するかは，基本場の温度及び水蒸気の垂直分布に依存している。

その卓越波長が数百キロ以下で生長率が $10^{-5} \sim 10^{-6} \mathrm{sec}^{-1}$ 程度の台風スケールの擾乱は mode (H) あるいは mode HM) に属している。台風スケールの擾乱は基本場の下層の安定度が小さい時又は下層が湿潤である時発達しやす い。こうした擾乱の発達に対して，対流圈下層の積雲対流による熱放出がきわめて重要な役割を果す。

最後に上述の台風スケールの擾乱とOoyama（1964）により提出された台風モードとの比較を試みた。 